\title{
Extinction and Persistence in Mean of a Novel Delay Impulsive Stochastic Infected Predator-Prey System with Jumps
}

\author{
Guodong Liu, ${ }^{1}$ Xiaohong Wang, ${ }^{1}$ Xinzhu Meng, ${ }^{1,2,3}$ and Shujing Gao ${ }^{3}$ \\ ${ }^{1}$ College of Mathematics and Systems Science, Shandong University of Science and Technology, Qingdao 266590, China \\ ${ }^{2}$ State Key Laboratory of Mining Disaster Prevention and Control Co-founded by Shandong Province and the Ministry of \\ Science and Technology, Shandong University of Science and Technology, Qingdao 266590, China \\ ${ }^{3}$ Key Laboratory of Jiangxi Province for Numerical Simulation and Emulation Techniques, Gannan Normal University, \\ Ganzhou 341000, China \\ Correspondence should be addressed to Xinzhu Meng; mxz721106@sdust.edu.cn
}

Received 25 March 2017; Revised 24 April 2017; Accepted 4 May 2017; Published 20 June 2017

Academic Editor: Fathalla A. Rihan

Copyright (C) 2017 Guodong Liu et al. This is an open access article distributed under the Creative Commons Attribution License, which permits unrestricted use, distribution, and reproduction in any medium, provided the original work is properly cited.

In this paper, we explore an impulsive stochastic infected predator-prey system with Lévy jumps and delays. The main aim of this paper is to investigate the effects of time delays and impulse stochastic interference on dynamics of the predator-prey model. First, we prove some properties of the subsystem of the system. Second, in view of comparison theorem and limit superior theory, we obtain the sufficient conditions for the extinction of this system. Furthermore, persistence in mean of the system is also investigated by using the theory of impulsive stochastic differential equations (ISDE) and delay differential equations (DDE). Finally, we carry out some simulations to verify our main results and explain the biological implications.

\section{Introduction}

With the development of the economy, environmental pollution is caused by various industries and other activities of human, which has been one of the most important social problems in the world today. Many species have gone extinct due to the toxicant in the environment. Therefore, controlling the environmental pollution has been the important topics around the world. There are many researchers which have investigated the pollution models in recent years [1-3]. In addition, a lot of animal populations suffer from infectious disease, so some scholars investigated the predator-prey systems with diseases [4-8]. For example, a deterministic predator-prey model with infected predator in an impulsive polluted environment is described by the following equation:

$$
\begin{aligned}
& \mathrm{d} X(t)=X(t)\left[r-\delta_{1} c_{1}(t)-a_{11} X(t)-a_{12} S(t)\right] \mathrm{d} t, \\
& \mathrm{~d} S(t)=S(t)
\end{aligned}
$$$$
\cdot\left[-\mu_{1}-\delta_{2} \varsigma_{2}(t)+a_{21} X(t)-\beta I(t)-a_{22} S(t)\right] \mathrm{d} t,
$$

$$
\begin{aligned}
& \mathrm{d} I(t)=I(t)\left[-\mu_{2}-\delta_{3} c_{3}(t)+\beta S(t)-a_{33} I(t)\right] \mathrm{d} t, \\
& \dot{c}_{1}(t)=k_{1} c_{e}(t)-g_{1} c_{1}(t)-m_{1} c_{1}(t), \\
& \dot{c}_{2}(t)=k_{2} c_{e}(t)-g_{2} c_{2}(t)-m_{2} c_{2}(t), \\
& \dot{c}_{3}(t)=k_{3} c_{e}(t)-g_{3} c_{3}(t)-m_{3} c_{3}(t), \\
& \dot{c}_{e}(t)=-h c_{e}(t)
\end{aligned}
$$$$
t \neq n T \text {, }
$$

$$
\begin{aligned}
& \Delta X(t)=0, \\
& \Delta S(t)=0, \\
& \Delta I(t)=0, \\
& \Delta c_{i}(t)=0, \\
& \Delta c_{e}(t)=u,
\end{aligned}
$$

$$
i=1,2,3, t=n T, n \in \mathbb{Z}^{+},
$$


where $\Delta X(t)=X\left(t^{+}\right)-X(t), \Delta S(t)=S\left(t^{+}\right)-S(t), \Delta I(t)=$ $I\left(t^{+}\right)-I(t), \Delta c_{i}(t)=c_{i}\left(t^{+}\right)-c_{i}(t), \Delta c_{e}(t)=c_{e}\left(t^{+}\right)-c_{e}(t)$, $X(t)$ is the density of the prey at time $t$, and $S(t)$ and $I(t)$ represent the density of susceptible predator and infected predator at time $t$, respectively. $c_{i}(t)(i=1,2,3)$ and $c_{e}(t)$ stand for the concentrations of the toxicant in the organism and the environment at time $t$, respectively. $\delta_{i}(i=1,2,3)$ are dose-response parameter to the toxicant. $r$ stands for the intrinsic growth rate of the prey. $\mu_{i}(i=1,2)$ denote the death rates of $S(t)$ and $I(t)$, respectively. $\beta$ is the infection rate. $a_{i i}(i=1,2,3)$ are density-dependent coefficients, and $a_{12}$ and $a_{21}$ represent predation rate and ingestion rate, respectively. $k_{i}(i=1,2,3)$ are environmental toxicant uptake rates, $m_{i}(i=1,2,3)$ denote organismal net depuration rates, $g_{i}(i=1,2,3)$ represent organismal net ingestion rates, $h$ is the loss rate of toxicant from environment, and $u$ denotes the amount of pulsed input concentration of the toxicant at every time. The above parameters are all positive constants. Next, we propose a new mathematical model by taking more factors into account based on model (1).

In the natural world, time delay often occurs in almost every situation. Thus it is significant to take time delay into consideration [9-14]. As we know, deterministic model is not enough to describe the species activities. Sometimes, the species activities may be disturbed by environmental noises. May [15] revealed that the birth rates, death rates, carrying capacities, competition coefficients, and other parameters involved in the system should exhibit random fluctuation to a greater or lesser extent. Hence some parameters should be stochastic [16-26]. First, we assume that the intrinsic growth rate and the death rates of species are disturbed by white noise, then $r$ and $\mu_{i}$ can be replaced by

$$
\begin{gathered}
r \longrightarrow r+\sigma_{1} \dot{B}_{1}(t), \\
-\mu_{1} \longrightarrow-\mu_{1}+\sigma_{2} \dot{B}_{2}(t), \\
-\mu_{2} \longrightarrow-\mu_{2}+\sigma_{3} \dot{B}_{3}(t),
\end{gathered}
$$

where $B_{i}(t)(i=1,2,3)$ are standard Brownian motions and $\sigma_{i}$ are the intensities of $B_{i}(t) . B_{i}(t)$ are mutually independent defined on a complete probability space $\left(\Omega, \mathscr{F},\{\mathscr{F}\}_{t \geq 0}, \mathscr{P}\right)$.

Furthermore, populations may suffer from sudden environmental fluctuations, such as floods and earthquakes, which cannot be described by Brownian motions. To explain these phenomena, introducing a jump process into the underlying population dynamics is one of the important methods. Thus, there are many scholars introduce Lévy jumps into the population system [27-31]. Taking all above influences into consideration, we focus on the infected stochastic predatorprey system with Lévy jumps and delays in a polluted environment

$$
\begin{aligned}
& \mathrm{d} X(t)=X\left(t^{-}\right)\left[r-\delta_{1} c_{1}(t)-a_{11} X\left(t^{-}\right)\right. \\
& \left.\quad-a_{12} e^{-d_{1} \tau_{1}} S\left(t^{-}-\tau_{1}\right)\right] \mathrm{d} t+\sigma_{1} X(t) \mathrm{d} B_{1}(t) \\
& \quad+\int_{\mathbb{Y}} X\left(t^{-}\right) \gamma_{1}(u) \widetilde{N}(\mathrm{~d} t, \mathrm{~d} u),
\end{aligned}
$$

$$
\begin{aligned}
& \mathrm{d} S(t)=S\left(t^{-}\right)\left[-\mu_{1}-\delta_{2} c_{2}(t)+a_{21} e^{-d_{2} \tau_{2}} X\left(t^{-}-\tau_{2}\right)\right. \\
& \left.-\beta e^{-d_{3} \tau_{3}} I\left(t^{-}-\tau_{3}\right)-a_{22} S\left(t^{-}\right)\right] \mathrm{d} t \\
& +\sigma_{2} S(t) \mathrm{d} B_{2}(t)+\int_{\mathbb{Y}} S\left(t^{-}\right) \gamma_{2}(u) \widetilde{N}(\mathrm{~d} t, \mathrm{~d} u), \\
& \mathrm{d} I(t)=I\left(t^{-}\right)\left[-\mu_{2}-\delta_{3} c_{3}(t)+\beta e^{-d_{4} \tau_{4}} S\left(t^{-}-\tau_{4}\right)\right. \\
& \left.\quad-a_{33} I\left(t^{-}\right)\right] \mathrm{d} t+\sigma_{3} I(t) \mathrm{d} B_{3}(t) \\
& +\int_{\mathbb{Y}} I\left(t^{-}\right) \gamma_{3}(u) \widetilde{N}(\mathrm{~d} t, \mathrm{~d} u), \\
& \dot{c}_{1}(t)=k_{1} c_{e}(t)-g_{1} c_{1}(t)-m_{1} c_{1}(t), \\
& \dot{c}_{2}(t)=k_{2} c_{e}(t)-g_{2} c_{2}(t)-m_{2} c_{2}(t), \\
& \dot{c}_{3}(t)=k_{3} c_{e}(t)-g_{3} c_{3}(t)-m_{3} c_{3}(t), \\
& \dot{c}_{e}(t)=-h c_{e}(t),
\end{aligned}
$$

$$
\begin{aligned}
& \Delta X(t)=0, \\
& \Delta S(t)=0, \\
& \Delta I(t)=0, \\
& \Delta c_{i}(t)=0, \\
& \Delta c_{e}(t)=u,
\end{aligned}
$$

$$
i=1,2,3, t=n T, n \in \mathbb{Z}^{+},
$$

where $X\left(t^{-}\right), S\left(t^{-}\right)$, and $I\left(t^{-}\right)$stand for the left limits of $X(t)$, $S(t)$, and $I(t)$, respectively. $N(\mathrm{~d} t, \mathrm{~d} u)$ denotes a Poisson counting measure with characteristic measure $v$ which defines a measurable bounded subset $\mathbb{Y}$ of $(0, \infty)$ with $v(\mathbb{Y})<\infty$ and $\widetilde{N}(\mathrm{~d} t, \mathrm{~d} u)=N(\mathrm{~d} t, \mathrm{~d} u)-v(\mathrm{~d} u) \mathrm{d} t$, and $\gamma_{i}$ is bounded and continuous with respect to $\nu$ and is $\mathfrak{B}(\mathbb{Y}) \times \mathscr{F}_{t}$-measurable, and $1+\gamma_{i}>0(i=1,2,3)$ (see [27-30]). Moreover, $B_{i}(t)(i=$ $1,2,3)$ are independent of $N, d_{i}$ are death rates of species, and $\tau_{i} \geq 0(i=1,2,3,4)$ represent the time delay. Other parameters are defined as system (1).

The rest of this paper is arranged as follows. Section 2 introduces some lemmas which will be used in our main results. In Section 3, we show the main results. We examine the extinction of system (3) in Section 3.1; in Section 3.2 we also prove the permanence in mean of this system. Finally, we present some simulations and conclusions in Section 4.

\section{Preliminary Results}

Throughout the paper, we assume that $X(t), S(t), I(t)$, and $c_{i}(t)$ are continuous at $t=n T$ and $c_{e}(t)$ is left continuous at $t=n T$ and $c_{e}\left(n T^{+}\right)=\lim _{t \rightarrow n T^{+}} c_{e}(t)$. Moreover, let $\left(\Omega, \mathscr{F},\{\mathscr{F}\}_{t \geq 0}, \mathscr{P}\right)$ be a complete probability space with a 
filtration $\left\{\mathscr{F}_{t}\right\}_{t \geq 0}$ satisfying the common conditions (i.e., it is increasing and right continuous while $\mathscr{F}_{0}$ contains all $\mathscr{P}$-null sets).

For the sake of convenience, we introduce some notions and some lemmas which will be used for the main results. We define

$$
\begin{aligned}
& \tau=\max \left\{\tau_{1}, \tau_{2}, \tau_{3}, \tau_{4}\right\}, \\
& b_{1}=r-\frac{1}{2} \sigma_{1}^{2} \\
& -\int_{\mathbb{Y}}\left(\gamma_{1}(u)-\ln \left(1+\gamma_{1}(u)\right)\right) v(\mathrm{~d} u), \\
& b_{2}=\mu_{1}+\frac{1}{2} \sigma_{2}^{2} \\
& +\int_{\mathbb{Y}}\left(\gamma_{2}(u)-\ln \left(1+\gamma_{2}(u)\right)\right) \nu(\mathrm{d} u), \\
& b_{3}=\mu_{2}+\frac{1}{2} \sigma_{3}^{2} \\
& +\int_{\mathbb{Y}}\left(\gamma_{3}(u)-\ln \left(1+\gamma_{3}(u)\right)\right) \nu(\mathrm{d} u), \\
& \Delta_{1}=\left(b_{1}-\delta_{1} \bar{c}_{1}\right) a_{21} e^{-d_{2} \tau_{2}}-\left(b_{2}+\delta_{2} \bar{c}_{2}\right) a_{11}, \\
& \Delta^{\prime}=a_{11} a_{22}+a_{12} a_{21} e^{-d_{1} \tau_{1}-d_{2} \tau_{2}}, \\
& \Delta_{2}=\left(b_{1}-\delta_{1} \bar{c}_{1}\right)\left(a_{22} a_{33}+\beta^{2} e^{-d_{3} \tau_{3}-d_{4} \tau_{4}}\right) \\
& +\left(b_{2}+\delta_{2} \bar{c}_{2}\right) a_{33} a_{12} e^{-d_{1} \tau_{1}} \\
& -\left(b_{3}+\delta_{3} \bar{c}_{3}\right) a_{12} \beta e^{-d_{1} \tau_{1}-d_{3} \tau_{3}}, \\
& \Delta_{3}=a_{33} \Delta_{1}+\left(b_{3}+\delta_{3} \bar{c}_{3}\right) a_{11} \beta e^{-d_{3} \tau_{3}}, \\
& \Delta_{4}=\beta e^{-d_{4} \tau_{4}} \Delta_{1}-\left(b_{3}+\delta_{3} \bar{c}_{3}\right) \Delta^{\prime} \text {, } \\
& \Delta=a_{33} \Delta^{\prime}+a_{11} \beta^{2} e^{-\left(d_{3} \tau_{3}+d_{4} \tau_{4}\right)}, \\
& \mathscr{R}=\frac{\beta e^{-d_{4} \tau_{4}} \Delta_{1}}{\left(b_{3}+\delta_{3} \bar{c}_{3}\right) a_{11} a_{22}}, \\
& \langle f(t)\rangle=\frac{1}{t} \int_{0}^{t} f(t) \mathrm{d} t, \\
& f^{*}=\limsup _{t \rightarrow+\infty} f(t), \\
& f_{*}=\liminf _{t \rightarrow+\infty} f(t),
\end{aligned}
$$

where $f(t)$ is a bounded continuous function on $[0,+\infty)$.

Then we show some basic properties of the subsystem of system (3)

$$
\begin{aligned}
& \dot{c}_{1}(t)=k_{1} c_{e}(t)-g_{1} c_{1}(t)-m_{1} c_{1}(t), \\
& \dot{c}_{2}(t)=k_{2} c_{e}(t)-g_{2} c_{2}(t)-m_{2} c_{2}(t), \\
& \dot{c}_{3}(t)=k_{3} c_{e}(t)-g_{3} c_{3}(t)-m_{3} c_{3}(t),
\end{aligned}
$$

$$
\dot{c}_{e}(t)=-h c_{e}(t)
$$

$$
t \neq n T, n \in Z^{+}
$$

$$
\begin{aligned}
& \Delta c_{i}(t)=0, \\
& \Delta c_{e}(t)=u,
\end{aligned}
$$

$$
i=1,2,3, t=n T, n \in Z^{+} \text {. }
$$

Lemma 1 (see [3]). System (5) has a unique positive T-periodic solution $\left(\widetilde{c}_{1}(t), \widetilde{c}_{2}(t), \widetilde{c}_{3}(t), \widetilde{c}_{e}(t)\right)^{T}$ which is globally asymptotical stable. Furthermore, if $c_{i}(0)>\widetilde{c}_{i}(0)$ and $c_{e}(0)>\widetilde{c}_{e}(0)$, then $c_{i}(t)>\widetilde{c}_{i}(t)$ and $c_{e}(t)>\widetilde{c}_{e}(t)$ for all $t \geq 0$, where

$$
\begin{aligned}
\tilde{c}_{i}(t)= & \widetilde{c}_{i}(0) e^{-\left(g_{i}+m_{i}\right)(t-n T)} \\
& +\frac{k_{i} u\left(e^{-\left(g_{i}+m_{i}\right)(t-n T)}-e^{-h(t-n T)}\right)}{\left(h-g_{i}-m_{i}\right)\left(1-e^{-h T}\right)}, \\
\widetilde{c}_{e}(t)= & \frac{u e^{-h(t-n T)}}{1-e^{-h T}}, \\
\widetilde{c}_{i}(0)= & \frac{k_{i} u\left(e^{-\left(g_{i}+m_{i}\right) T}-e^{-h T}\right)}{\left(h-g_{i}-m_{i}\right)\left(1-e^{-\left(g_{i}+m_{i}\right) T}\right)\left(1-e^{-h T}\right)}, \\
\widetilde{c}_{e}(0)= & \frac{u}{1-e^{-h T}},
\end{aligned}
$$

for $t \in(n T,(n+1) T], i=1,2,3$, and $n \in Z^{+}$.

It can be obtained from a simple calculation that

$$
\int_{n T}^{(n+1) T} \tilde{c}_{i}(t) \mathrm{d} t=\frac{k_{i} u}{h\left(g_{i}+m_{i}\right)} .
$$

Since $\widetilde{c}_{i}(t)$ is a periodic function, then we get

$$
\begin{aligned}
\lim _{t \rightarrow \infty}\left\langle c_{i}(t)\right\rangle^{*} & \leq \lim _{n \rightarrow \infty} \frac{1}{n T} \int_{0}^{(n+1) T} \widetilde{c}_{i}(t) \mathrm{d} t \\
& =\lim _{n \rightarrow \infty} \frac{n+1}{n T} \int_{n T}^{(n+1) T} \widetilde{c}_{i}(t) \mathrm{d} t \\
& =\frac{k_{i} u}{h\left(g_{i}+m_{i}\right) T}, \\
\lim _{t \rightarrow \infty}\left\langle c_{i}(t)\right\rangle_{*} & \geq \lim _{n \rightarrow \infty} \frac{1}{(n+1) T} \int_{0}^{n T} \widetilde{c}_{i}(t) \mathrm{d} t \\
& =\lim _{n \rightarrow \infty} \frac{n}{(n+1) T} \int_{(n-1) T}^{n T} \widetilde{c}_{i}(t) \mathrm{d} t \\
& =\frac{k_{i} u}{h\left(g_{i}+m_{i}\right) T} .
\end{aligned}
$$

Thus we have

$$
\lim _{t \rightarrow+\infty}\left\langle c_{i}(t)\right\rangle=\frac{k_{i} u}{h\left(g_{i}+m_{i}\right) T}:=\bar{c}_{i} .
$$


From Lemma 1, we know that the long time dynamical behaviors of system (3) can be replaced by the dynamical behaviors of the following limiting system:

$$
\begin{aligned}
& \mathrm{d} X(t)=X\left(t^{-}\right)\left[r-\delta_{1} \widetilde{c}_{1}(t)-a_{11} X\left(t^{-}\right)\right. \\
& \left.\quad-a_{12} e^{-d_{1} \tau_{1}} S\left(t^{-}-\tau_{1}\right)\right] \mathrm{d} t+\sigma_{1} X(t) \mathrm{d} B_{1}(t) \\
& \quad+\int_{\mathbb{V}} X\left(t^{-}\right) \gamma_{1}(u) \widetilde{N}(\mathrm{~d} t, \mathrm{~d} u), \\
& \quad \mathrm{d} S(t)=S\left(t^{-}\right)\left[-\mu_{1}-\delta_{2} \widetilde{\mathcal{c}}_{2}(t)+a_{21} e^{-d_{2} \tau_{2}} X\left(t^{-}-\tau_{2}\right)\right. \\
& \left.\quad+\beta e^{-d_{3} \tau_{3}} I\left(t^{-}-\tau_{3}\right)-a_{22} S\left(t^{-}\right)\right] \mathrm{d} t \\
& \quad+\sigma_{2} S(t) \mathrm{d} B_{2}(t) \\
& \quad+\int_{\mathbb{Y}} S\left(t^{-}\right) \gamma_{2}(u) \widetilde{N}(\mathrm{~d} t, \mathrm{~d} u), \\
& \mathrm{d} I(t)=I\left(t^{-}\right)\left[-\mu_{2}-\delta_{3} \widetilde{c}_{3}(t)+\beta e^{-d_{4} \tau_{4}} S\left(t^{-}-\tau_{4}\right)\right. \\
& \left.\quad-a_{33} I\left(t^{-}\right)\right] \mathrm{d} t+\sigma_{3} I(t) \mathrm{d} B_{3}(t) \\
& \quad+\int_{\mathbb{Y}} I\left(t^{-}\right) \gamma_{3}(u) \widetilde{N}(\mathrm{~d} t, \mathrm{~d} u) .
\end{aligned}
$$

Now we give an assumption which will be used in the following proof.

Assumption 2. There exist constants $C_{i}$ such that

$$
\int_{\mathbb{Y}}\left[\gamma_{i}-\ln \left(1+\gamma_{i}\right)\right] \nu(\mathrm{d} u) \leq C_{i}, \quad(i=1,2,3) .
$$

Lemma 3. For any given initial value $\left(\left(\phi_{1}(t), \phi_{2}(t), \phi_{3}(t)\right)\right.$ : $\left.-\tau \leq t \leq 0, \tau=\max \left\{\tau_{1}, \tau_{2}, \tau_{3}, \tau_{4}\right\}\right) \in C\left([-\tau, 0] ; R_{+}^{3}\right)$, there is a unique solution $(X(t), S(t), I(t))$ of (10) on $t \geq \tau$ and the solution will remain in $\mathbb{R}_{+}^{2}$ with probability 1 .

Proof. This proof is the same as Theorem 3.1 in [11] by defining

$$
V(X, S, I)=V_{1}(X, S, I)+V_{2}(X, S, I)
$$

where

$$
\begin{aligned}
V_{1}(X, S, I)= & X(t)-1-\ln X(t)+S(t)-1-\ln S(t) \\
& +I(t)-1-\ln I(t) \\
V_{2}(X, S, I)= & a_{12} e^{-d_{1} \tau_{1}} \int_{t}^{t+\tau_{1}} S\left(s-\tau_{1}\right) \mathrm{d} s \\
& +\frac{1}{2} \int_{t}^{t+\tau_{2}} X^{2}\left(s-\tau_{2}\right) \mathrm{d} s \\
& +\beta e^{-d_{3} \tau_{3}} \int_{t}^{t+\tau_{3}} I\left(s-\tau_{3}\right) \mathrm{d} s \\
& +\frac{1}{2} \int_{t}^{t+\tau_{4}} S^{2}\left(s-\tau_{4}\right) \mathrm{d} s .
\end{aligned}
$$

Thus, we omit it here.
The stochastic comparison theorem and limit superior and limit inferior theory are given as follows.

Lemma 4 (see [27]). Suppose that $Y(t) \in C\left(\Omega \times[0, \infty), \mathbb{R}_{+}\right)$. that

(i) If there exist three positive constants $T, \lambda$, and $\lambda_{0}$ such

$$
\begin{aligned}
\ln Y(t) \leq & \lambda t-\lambda_{0} \int_{0}^{t} Y(s) \mathrm{d} s+\alpha B(t) \\
& +\sum_{i=1}^{n} \eta_{i} \int_{0}^{t} \int_{\mathbb{V}} \ln \left(1+\gamma_{i}(u)\right) \widetilde{N}(\mathrm{~d} s, \mathrm{~d} u) \quad \text { a.s. }
\end{aligned}
$$

for all $t \geq T$, where $\alpha$ and $\eta_{i}$ are constants, then

$$
\begin{gathered}
\limsup \langle Y(t)\rangle=\langle Y(t)\rangle^{*} \leq \frac{\lambda}{\lambda_{0}} \quad \text { a.s., if } \lambda \geq 0, \\
\lim _{t \rightarrow \infty} Y(t)=0 \quad \text { a.s., if } \lambda<0 .
\end{gathered}
$$
that

(ii) If there exist three positive constants $T, \lambda$, and $\lambda_{0}$ such

$$
\begin{aligned}
\ln Y(t) \geq & \lambda t-\lambda_{0} \int_{0}^{t} Y(s) \mathrm{d} s+\alpha B(t) \\
& +\sum_{i=1}^{n} \eta_{i} \int_{0}^{t} \int_{\mathbb{V}} \ln \left(1+\gamma_{i}(u)\right) \widetilde{N}(\mathrm{~d} s, \mathrm{~d} u) \quad \text { a.s. }
\end{aligned}
$$

for all $t \geq T$, then $\lim \inf _{t \rightarrow \infty}\langle Y(t)\rangle=\langle Y(t)\rangle_{*} \geq \lambda / \lambda_{0}$ a.s.

First, we explore the following auxiliary system:

$$
\begin{aligned}
& \mathrm{d} X_{1}(t)=X\left(t^{-}\right)\left[r-\delta_{1} \widetilde{c}_{1}(t)-a_{11} X_{1}\left(t^{-}\right)\right] \mathrm{d} t \\
& \quad+\sigma_{1} X_{1}(t) \mathrm{d} B_{1}(t) \\
& \quad+\int_{\mathbb{Y}} X_{1}\left(t^{-}\right) \gamma_{1}(u) \widetilde{N}(\mathrm{~d} t, \mathrm{~d} u), \\
& \mathrm{d} S_{1}(t)=S_{1}\left(t^{-}\right)\left[-\mu_{1}-\delta_{2} \widetilde{\mathcal{C}}_{2}(t)\right. \\
& \left.\quad+a_{21} e^{-d_{2} \tau_{2}} X_{1}\left(t^{-}-\tau_{2}\right)-a_{22} S_{1}\left(t^{-}\right)\right] \mathrm{d} t \\
& \quad+\sigma_{2} S_{1}(t) \mathrm{d} B_{2}(t) \\
& \quad+\int_{\mathbb{Y}} S_{1}\left(t^{-}\right) \gamma_{2}(u) \widetilde{N}(\mathrm{~d} t, \mathrm{~d} u), \\
& \mathrm{d} I_{1}(t)=I_{1}\left(t^{-}\right)\left[-\mu_{2}-\delta_{3} \widetilde{c}_{3}(t)+\beta e^{-d_{4} \tau_{4}} S_{1}\left(t^{-}-\tau_{4}\right)\right. \\
& \left.\quad-a_{33} I_{1}\left(t^{-}\right)\right] \mathrm{d} t+\sigma_{3} I_{1}(t) \mathrm{d} B_{3}(t) \\
& \quad+\int_{\mathbb{Y}} I_{1}\left(t^{-}\right) \gamma_{3}(u) \widetilde{N}(\mathrm{~d} t, \mathrm{~d} u) .
\end{aligned}
$$

Lemma 5. For system (17), let $\left(X_{1}(t), S_{1}(t), I_{1}(t)\right)$ be the solution of this system with initial value $\left(\left(\phi_{1}(t), \phi_{2}(t), \phi_{3}(t)\right)\right.$ : $-\tau \leq t \leq 0) \in C\left([-\tau, 0] ; R_{+}^{3}\right)$. 
(i) If $b_{1}<\delta_{1} \bar{c}_{1}$, then

$$
\begin{gathered}
\lim _{t \rightarrow \infty} X_{1}(t)=0, \\
\lim _{t \rightarrow \infty} S_{1}(t)=0, \\
\lim _{t \rightarrow \infty} I_{1}(t)=0,
\end{gathered}
$$

a.s.

(ii) If $b_{1}>\delta_{1} \bar{c}_{1}$, when $\Delta_{1}<0$, then

$$
\begin{aligned}
\lim _{t \rightarrow \infty}\left\langle X_{1}(t)\right\rangle & =\frac{b_{1}-\delta_{1} \bar{c}_{1}}{a_{11}}, \\
\lim _{t \rightarrow \infty} S_{1}(t) & =0, \\
\lim _{t \rightarrow \infty} I_{1}(t) & =0, \\
\lim _{t \rightarrow \infty} \frac{\ln X_{1}(t)}{t} & =0,
\end{aligned}
$$

a.s.

(iv) If $b_{1}>\delta_{1} \bar{c}_{1}$, when $\mathscr{R}>1$, then

$$
\begin{aligned}
& \lim _{t \rightarrow \infty}\left\langle X_{1}(t)\right\rangle=\frac{b_{1}-\delta_{1} \bar{c}_{1}}{a_{11}}, \\
& \lim _{t \rightarrow \infty}\left\langle S_{1}(t)\right\rangle=\frac{\Delta_{1}}{a_{11} a_{22}}, \\
& \lim _{t \rightarrow \infty}\left\langle I_{1}(t)\right\rangle=\frac{\beta e^{-d_{4} \tau_{4}} \Delta_{1}-\left(b_{3}+\delta_{3} \bar{c}_{3}\right) a_{11} a_{22}}{a_{11} a_{22} a_{33}}, \\
& \lim _{t \rightarrow \infty} \frac{\ln I_{1}(t)}{t}=0,
\end{aligned}
$$$$
\text { a.s, }
$$

a.s.

Proof. Applying Itô's formula to system (17) leads to

$$
\begin{aligned}
& \mathrm{d} \ln X_{1}=\left[b_{1}-\delta_{1} \widetilde{c}_{1}(t)-a_{11} X_{1}(t)\right] \mathrm{d} t+\sigma_{1} \mathrm{~d} B_{1}(t) \\
& +\int_{\mathbb{V}} \ln \left(1+\gamma_{1}(u)\right) \widetilde{N}(\mathrm{~d} t, \mathrm{~d} u), \\
& \mathrm{d} \ln S_{1}=\left[-b_{2}-\delta_{2} \widetilde{\mathcal{c}}_{2}(t)+a_{21} e^{-d_{2} \tau_{2}} X_{1}\left(t-\tau_{2}\right)\right. \\
& \left.\quad-a_{22} S_{1}(t)\right] \mathrm{d} t+\sigma_{2} \mathrm{~d} B_{2}(t) \\
& \quad+\int_{\mathbb{V}} \ln \left(1+\gamma_{2}(u)\right) \widetilde{N}(\mathrm{~d} t, \mathrm{~d} u),
\end{aligned}
$$

$$
\begin{aligned}
& \mathrm{d} \ln I_{1}=\left[-b_{3}-\delta_{3} \widetilde{\mathcal{S}}_{3}(t)+\beta e^{-d_{4} \tau_{4}} S_{1}\left(t-\tau_{4}\right)\right. \\
& \left.-a_{33} I_{1}(t)\right] \mathrm{d} t+\sigma_{3} \mathrm{~d} B_{3}(t) \\
& +\int_{\mathbb{V}} \ln \left(1+\gamma_{3}(u)\right) \widetilde{N}(\mathrm{~d} t, \mathrm{~d} u) .
\end{aligned}
$$

Integrating both sides of (22), we have

$$
\begin{aligned}
& \ln X_{1}(t)-\ln X_{1}(0)=b_{1} t-\delta_{1} \int_{0}^{t} \widetilde{c}_{1}(s) \mathrm{d} s \\
& \quad-a_{11} \int_{0}^{t} X_{1}(s) \mathrm{d} s+\sigma_{1} B_{1}(t) \\
& \quad+\int_{0}^{t} \int_{\mathbb{Y}} \ln \left(1+\gamma_{1}(u)\right) \widetilde{N}(\mathrm{~d} s, \mathrm{~d} u), \\
& \ln S_{1}(t)-\ln S_{1}(0)=-b_{2} t-\delta_{2} \int_{0}^{t} \widetilde{\mathcal{C}}_{2}(s) \mathrm{d} s \\
& +a_{21} e^{-d_{2} \tau_{2}} \int_{0}^{t} X_{1}\left(t-\tau_{2}\right)-a_{22} \int_{0}^{t} S_{1}(s) \mathrm{d} s \\
& \quad+\sigma_{2} B_{2}(t)+\int_{0}^{t} \int_{\mathbb{Y}} \ln \left(1+\gamma_{2}(u)\right) \widetilde{N}(\mathrm{~d} s, \mathrm{~d} u) \\
& \quad=-b_{2} t-\delta_{2} \int_{0}^{t} \widetilde{\mathcal{C}}_{2}(s) \mathrm{d} s+a_{21} e^{-d_{2} \tau_{2}} \int_{0}^{t} X_{1}(s) \mathrm{d} s \\
& \quad-a_{22} \int_{0}^{t} S_{1}(s) \mathrm{d} s \\
& \quad-a_{21} e^{-d_{2} \tau_{2}}\left(\int_{t}^{t+\tau_{2}} X_{1}\left(s-\tau_{2}\right) \mathrm{d} s\right. \\
& \left.\quad-\int_{0}^{\tau_{2}} X_{1}\left(s-\tau_{2}\right) \mathrm{d} s\right)+\sigma_{2} B_{2}(t) \\
& +\int_{0}^{t} \int_{\mathbb{Y}} \ln \left(1+\gamma_{2}(u)\right) \widetilde{N}(\mathrm{~d} s, \mathrm{~d} u),
\end{aligned}
$$

$$
\begin{aligned}
& \ln I_{1}(t)-\ln I_{1}(0)=-b_{3} t-\delta_{3} \int_{0}^{t} \widetilde{\mathcal{C}}_{3}(s) \mathrm{d} s \\
& +\beta e^{-d_{4} \tau_{4}} \int_{0}^{t} S_{1}\left(s-\tau_{4}\right) \mathrm{d} s-a_{33} \int_{0}^{t} I_{1}(s) \mathrm{d} s \\
& +\sigma_{3} B_{3}(t)+\int_{0}^{t} \int_{\mathbb{V}} \ln \left(1+\gamma_{3}(u)\right) \widetilde{N}(\mathrm{~d} s, \mathrm{~d} u)
\end{aligned}
$$$$
=-b_{3} t-\delta_{3} \int_{0}^{t} \widetilde{\mathcal{C}}_{3}(s) \mathrm{d} s+\beta e^{-d_{4} \tau_{4}} \int_{0}^{t} S_{1}(s) \mathrm{d} s
$$$$
-a_{33} \int_{0}^{t} I_{1}(s) \mathrm{d} s-\beta e^{-d_{4} \tau_{4}}\left(\int_{t}^{t+\tau_{4}} S_{1}\left(s-\tau_{4}\right) \mathrm{d} s\right.
$$$$
\left.-\int_{0}^{\tau_{4}} S_{1}\left(s-\tau_{4}\right) \mathrm{d} s\right)+\sigma_{3} B_{3}(t)
$$

$+\int_{0}^{t} \int_{\mathbb{V}} \ln \left(1+\gamma_{3}(u)\right) \widetilde{N}(\mathrm{~d} s, \mathrm{~d} u)$. 
Then we can obtain

$$
\begin{aligned}
& \frac{\ln X_{1}(t)-\ln X_{1}(0)}{t}=b_{1}-\delta_{1}\left\langle\widetilde{c}_{1}(t)\right\rangle-a_{11}\left\langle X_{1}(t)\right\rangle \\
& +\frac{\sigma_{1} B_{1}(t)}{t}+\frac{1}{t} \int_{0}^{t} \int_{\mathbb{Y}} \ln \left(1+\gamma_{1}(u)\right) \widetilde{N}(\mathrm{~d} s, \mathrm{~d} u), \\
& \frac{\ln S_{1}(t)-\ln S_{1}(0)}{t}+a_{21} e^{-d_{2} \tau_{2}} \\
& \cdot \frac{\left(\int_{t}^{t+\tau_{2}} X_{1}\left(s-\tau_{2}\right) \mathrm{d} s-\int_{0}^{\tau_{2}} X_{1}\left(s-\tau_{2}\right) \mathrm{d} s\right)}{t}=-b_{2} \\
& -\delta_{2}\left\langle\widetilde{c}_{2}(t)\right\rangle+a_{21} e^{-d_{2} \tau_{2}}\left\langle X_{1}(t)\right\rangle-a_{22}\left\langle S_{1}(t)\right\rangle \\
& +\frac{\sigma_{2} B_{2}(t)}{t}+\frac{1}{t} \int_{0}^{t} \int_{\mathbb{Y}} \ln \left(1+\gamma_{2}(u)\right) \widetilde{N}(\mathrm{~d} s, \mathrm{~d} u), \\
& \frac{\ln I_{1}(t)-\ln I_{1}(0)}{t}+\beta e^{-d_{4} \tau_{4}} \\
& +\frac{\sigma_{3} B_{3}(t)}{t}+\frac{1}{t} \int_{0}^{t} \int_{\mathbb{Y}} \ln \left(1+\gamma_{3}(u)\right) \widetilde{N}(\mathrm{~d} s, \mathrm{~d} u) . \\
& \quad \frac{\left(\int_{t}^{t+\tau_{4}} S_{1}\left(s-\tau_{4}\right) \mathrm{d} s-\int_{0}^{\tau_{4}} S_{1}\left(s-\tau_{4}\right) \mathrm{d} s\right)}{t}=-b_{3} \\
& \quad \delta_{3}\left\langle\widetilde{c}_{3}(t)\right\rangle+\beta e^{-d_{4} \tau_{4}}\left\langle S_{1}(t)\right\rangle-a_{33}\left\langle I_{1}(t)\right\rangle
\end{aligned}
$$

Case (i). From Lemma 4 and (24), if $b_{1}<\delta_{1} \bar{c}_{1}$, then we have

$$
\lim _{t \rightarrow \infty} X_{1}(t)=0 \quad \text { a.s. }
$$

Obviously, we find

$$
\begin{aligned}
& \lim _{t \rightarrow \infty} S_{1}(t)=0, \\
& \lim _{t \rightarrow \infty} I_{1}(t)=0,
\end{aligned}
$$

a.s.

Case (ii). By Lemma 4, it is derived from (24) and conditions that

$$
\begin{aligned}
\left\langle X_{1}(t)\right\rangle^{*} \leq & \frac{b_{1}-\delta_{1}\left\langle\widetilde{c}_{1}(t)\right\rangle_{*}}{a_{11}}, \\
\left\langle X_{1}(t)\right\rangle_{*} & \geq \frac{b_{1}-\delta_{1}\left\langle\widetilde{c}_{1}(t)\right\rangle^{*}}{a_{11}} .
\end{aligned}
$$

Since

$$
\lim _{t \rightarrow \infty}\left\langle\widetilde{c}_{i}(t)\right\rangle_{*}=\lim _{t \rightarrow \infty}\left\langle\widetilde{c}_{i}(t)\right\rangle^{*}=\bar{c}_{i}, \quad i=1,2,3,
$$

then we obtain

$$
\lim _{t \rightarrow \infty}\left\langle X_{1}(t)\right\rangle=\frac{b_{1}-\delta_{1} \bar{c}_{1}}{a_{11}} .
$$

Using Assumption 2 and the strong law of large numbers for local martingales, one has

$$
\begin{aligned}
& \lim _{t \rightarrow+\infty} \frac{1}{t} \int_{0}^{t} \int_{\mathbb{Y}} \ln \left(1+\gamma_{i}(u)\right) \widetilde{N}(\mathrm{~d} s, \mathrm{~d} u)=0, \\
& \lim _{t \rightarrow+\infty} \frac{\sigma_{i} B_{i}(t)}{t}=0, \\
& \text { a.s, } i=1,2,3 .
\end{aligned}
$$

Then, substituting (31) into (24) yields

$$
\lim _{t \rightarrow \infty} \frac{\ln X_{1}(t)}{t}=0 \text {, a.s. }
$$

Since

$$
\begin{gathered}
\lim _{t \rightarrow \infty} \frac{\int_{t}^{t+\tau_{2}} X_{1}\left(s-\tau_{2}\right) \mathrm{d} s}{t}=\lim _{t \rightarrow \infty} \frac{1}{t} \int_{t-\tau_{2}}^{t} X_{1}(s) \mathrm{d} s=0 \\
\lim _{t \rightarrow \infty} \frac{\int_{0}^{\tau_{2}} X_{1}\left(s-\tau_{2}\right) \mathrm{d} s}{t}=\lim _{t \rightarrow \infty} \frac{\int_{-\tau_{2}}^{0} X_{1}(s) \mathrm{d} s}{t}=0
\end{gathered}
$$

then we have that, for any $0<\varepsilon_{1}<\Delta_{1}$, there exists $T_{1}>0$ such that

$$
\begin{aligned}
&-\varepsilon_{1}<a_{21} e^{-d_{2} \tau_{2}} \\
& \cdot \frac{\left(\int_{t}^{t+\tau_{2}} X_{1}\left(s-\tau_{2}\right) \mathrm{d} s-\int_{0}^{\tau_{2}} X_{1}\left(s-\tau_{2}\right) \mathrm{d} s\right)}{t}<\varepsilon_{1}, \\
& t \geq T_{1} .
\end{aligned}
$$

Combining (25) with (36) yields

$$
\begin{aligned}
& \frac{\ln S_{1}(t)-\ln S_{1}(0)}{t} \\
& \leq-b_{2}-\delta_{2}\left\langle\widetilde{c}_{2}(t)\right\rangle_{*}+a_{21} e^{-d_{2} \tau_{2}}\left\langle X_{1}(t)\right\rangle^{*}+\varepsilon_{1} \\
& \quad-a_{22}\left\langle S_{1}(t)\right\rangle+\frac{\sigma_{2} B_{2}(t)}{t} \\
& \quad+\frac{1}{t} \int_{0}^{t} \int_{\mathbb{V}} \ln \left(1+\gamma_{2}(u)\right) \widetilde{N}(\mathrm{~d} s, \mathrm{~d} u) .
\end{aligned}
$$

From Lemma 4 and conditions, we have

$$
\lim _{t \rightarrow \infty} S_{1}(t)=0 \quad \text { a.s. }
$$

Obviously, we have

$$
\lim _{t \rightarrow \infty} I_{1}(t)=0 \quad \text { a.s. }
$$


Case (iii). By (25), we obtain

$$
\begin{aligned}
& \frac{\ln S_{1}(t)-\ln S_{1}(0)}{t} \\
& \geq-b_{2}-\delta_{2}\left\langle\widetilde{c}_{2}(t)\right\rangle^{*}+a_{21} e^{-d_{2} \tau_{2}}\left\langle X_{1}(t)\right\rangle_{*}-\varepsilon_{1} \\
& \quad-a_{22}\left\langle S_{1}(t)\right\rangle+\frac{\sigma_{2} B_{2}(t)}{t} \\
& \quad+\frac{1}{t} \int_{0}^{t} \int_{\mathbb{Y}} \ln \left(1+\gamma_{2}(u)\right) \widetilde{N}(\mathrm{~d} s, \mathrm{~d} u) .
\end{aligned}
$$

It can infer from (37), (40), and Lemma 4 that

$$
\begin{aligned}
& \left\langle S_{1}(t)\right\rangle^{*} \leq \frac{\Delta_{1}+\varepsilon_{1}}{a_{11} a_{22}}, \\
& \left\langle S_{1}(t)\right\rangle_{*} \geq \frac{\Delta_{1}-\varepsilon_{1}}{a_{11} a_{22}} .
\end{aligned}
$$

Since $\varepsilon_{1}$ is an arbitrary number, then we get

$$
\lim _{t \rightarrow \infty}\left\langle S_{1}(t)\right\rangle=\frac{\Delta_{1}}{a_{11} a_{22}} \text { a.s. }
$$

Combining this equality with (25) leads to

$$
\lim _{t \rightarrow \infty} \frac{\ln S_{1}(t)}{t}=0, \quad \text { a.s. }
$$

Similar to (34) and (35), we have that, for any $0<\varepsilon_{2}<$ $\beta e^{-d_{4} \tau_{4}} \Delta_{1}-\left(b_{3}+\delta_{3} \bar{c}_{3}\right) a_{11} a_{22}$, there exists $T_{2}>0$ such that

$$
\begin{aligned}
& \frac{\ln I_{1}(t)-\ln I_{1}(0)}{t} \\
& \leq-b_{3}-\delta_{3}\left\langle\widetilde{c}_{3}(t)\right\rangle_{*}+\beta e^{-d_{4} \tau_{4}}\left\langle S_{1}(t)\right\rangle^{*}+\varepsilon_{2} \\
& \quad-a_{33}\left\langle I_{1}(t)\right\rangle+\frac{\sigma_{3} B_{3}(t)}{t} \\
& \quad+\frac{1}{t} \int_{0}^{t} \int_{\mathbb{Y}} \ln \left(1+\gamma_{3}(u)\right) \widetilde{N}(\mathrm{~d} s, \mathrm{~d} u) .
\end{aligned}
$$

Note that $\mathscr{R}<1$, so we have

$$
\lim _{t \rightarrow \infty} I_{1}(t)=0 \quad \text { a.s. }
$$

Case (iv). By (26), we get

$$
\begin{aligned}
& \frac{\ln I_{1}(t)-\ln I_{1}(0)}{t} \\
& \geq-b_{3}-\delta_{3}\left\langle\widetilde{c}_{3}(t)\right\rangle^{*}+\beta e^{-d_{4} \tau_{4}}\left\langle S_{1}(t)\right\rangle_{*}-\varepsilon_{2} \\
& \quad-a_{33}\left\langle I_{1}(t)\right\rangle+\frac{\sigma_{3} B_{3}(t)}{t} \\
& \quad+\frac{1}{t} \int_{0}^{t} \int_{\mathbb{Y}} \ln \left(1+\gamma_{3}(u)\right) \widetilde{N}(\mathrm{~d} s, \mathrm{~d} u) .
\end{aligned}
$$

When $\mathscr{R}>1$, from (44) and (46), using Lemma 4 results in

$$
\begin{aligned}
& \left\langle I_{1}(t)\right\rangle^{*} \\
& \leq \frac{\beta e^{-d_{4} \tau_{4}} \Delta_{1}-\left(b_{3}+\delta_{3}\left\langle\widetilde{c}_{3}(t)\right\rangle_{*}\right) a_{11} a_{22}+\varepsilon_{2}}{a_{11} a_{22} a_{33}}, \\
& \left\langle I_{1}(t)\right\rangle_{*} \\
& \geq \frac{\beta e^{-d_{4} \tau_{4}} \Delta_{1}-\left(b_{3}+\delta_{3}\left\langle\widetilde{c}_{3}(t)\right\rangle^{*}\right) a_{11} a_{22}-\varepsilon_{2}}{a_{11} a_{22} a_{33}} .
\end{aligned}
$$

Since $\varepsilon_{2}$ is an arbitrary number, then we can obtain

$$
\lim _{t \rightarrow \infty}\left\langle I_{1}(t)\right\rangle=\frac{\beta e^{-d_{4} \tau_{4}} \Delta_{1}-\left(b_{3}+\delta_{3} \bar{c}_{3}\right) a_{11} a_{22}}{a_{11} a_{22} a_{33}} \text { a.s. }
$$

Combining this equality with (26) leads to

$$
\lim _{t \rightarrow \infty} \frac{\ln I_{1}(t)}{t}=0, \quad \text { a.s. }
$$

This completes the proof of Lemma 1.

\section{Main Results}

3.1. Extinction. Now we are going to show our main results. By Lemma 5, we can get the extinction of system (10).

Theorem 6. For system (10), let $(X(t), S(t), I(t))$ be the solution of this system with initial value $\left(\left(\phi_{1}(t), \phi_{2}(t), \phi_{3}(t)\right):-\tau \leq\right.$ $t \leq 0) \in C\left([-\tau, 0] ; R_{+}^{3}\right)$.

(i) If $b_{1}<\delta_{1} \bar{c}_{1}$, then

$$
\begin{gathered}
\lim _{t \rightarrow \infty} X(t)=0, \\
\lim _{t \rightarrow \infty} S(t)=0, \\
\lim _{t \rightarrow \infty} I(t)=0,
\end{gathered}
$$

a.s.

(ii) If $b_{1}>\delta_{1} \bar{c}_{1}$, when $\Delta_{1}<0$, then

$$
\begin{aligned}
\lim _{t \rightarrow \infty}\langle X(t)\rangle & =\frac{b_{1}-\delta_{1} \bar{c}_{1}}{a_{11}}, \\
\lim _{t \rightarrow \infty} S(t) & =0 \\
\lim _{t \rightarrow \infty} I(t) & =0
\end{aligned}
$$

a.s.

(iii) If $b_{1}>\delta_{1} \bar{c}_{1}, \Delta_{1}>0$, when $\mathscr{R}<1$, then

$$
\lim _{t \rightarrow \infty}\langle X(t)\rangle=\frac{\left(b_{1}-\delta_{1} \bar{c}_{1}\right) a_{22}+\left(b_{2}+\delta_{2} \bar{c}_{2}\right) a_{12} e^{-d_{1} \tau_{1}}}{\Delta^{\prime}}
$$

$$
\begin{gathered}
\lim _{t \rightarrow \infty}\langle S(t)\rangle=\frac{\Delta_{1}}{\Delta^{\prime}} \quad \text { a.s, } \\
\lim _{t \rightarrow \infty} I(t)=0 \quad \text { a.s. }
\end{gathered}
$$


Proof. By stochastic comparison theorem, we have

$$
\begin{aligned}
& X(t) \leq X_{1}(t), \\
& S(t) \leq S_{1}(t), \\
& I(t) \leq I_{1}(t) .
\end{aligned}
$$

By (34), we derive

$$
\lim _{t \rightarrow \infty} \frac{\int_{t}^{t+\tau_{2}} X\left(s-\tau_{2}\right) \mathrm{d} s}{t}=0
$$

In the same way, we can verify that

$$
\begin{aligned}
\lim _{t \rightarrow \infty} \frac{\int_{t}^{t+\tau_{2}} S\left(s-\tau_{2}\right) \mathrm{d} s}{t} & =0, \\
\lim _{t \rightarrow \infty} \frac{\int_{t}^{t+\tau_{2}} I\left(s-\tau_{2}\right) \mathrm{d} s}{t} & =0 .
\end{aligned}
$$

It follows from (33), (43), and (49) that

$$
\begin{aligned}
& {\left[\frac{\ln X(t)}{t}\right]^{*} \leq \lim _{t \rightarrow \infty} \frac{\ln X_{1}(t)}{t}=0,} \\
& {\left[\frac{\ln S(t)}{t}\right]^{*} \leq \lim _{t \rightarrow \infty} \frac{\ln S_{1}(t)}{t}=0,} \\
& {\left[\frac{\ln I(t)}{t}\right]^{*} \leq \lim _{t \rightarrow \infty} \frac{\ln I_{1}(t)}{t}=0 .}
\end{aligned}
$$

Applying Itô's formula to system (10) leads to

$$
\begin{aligned}
& \mathrm{d} \ln X=\left[b_{1}-\delta_{1} \widetilde{c}_{1}(t)-a_{11} X(t)\right. \\
& \left.\quad-a_{12} e^{-d_{1} \tau_{1}} S\left(t-\tau_{1}\right)\right] \mathrm{d} t+\sigma_{1} \mathrm{~d} B_{1}(t) \\
& \quad+\int_{\mathbb{Y}} \ln \left(1+\gamma_{1}(u)\right) \widetilde{N}(\mathrm{~d} t, \mathrm{~d} u), \\
& \mathrm{d} \ln S=\left[-b_{2}-\delta_{2} \widetilde{c}_{2}(t)+a_{21} e^{-d_{2} \tau_{2}} X\left(t-\tau_{2}\right)\right. \\
& \left.\quad-\beta e^{-d_{3} \tau_{3}} I\left(t-\tau_{3}\right)-a_{22} S(t)\right] \mathrm{d} t+\sigma_{2} \mathrm{~d} B_{2}(t) \\
& \quad+\int_{\mathbb{Y}} \ln \left(1+\gamma_{2}(u)\right) \widetilde{N}(\mathrm{~d} t, \mathrm{~d} u), \\
& \mathrm{d} \ln I=\left[-b_{3}-\delta_{3} \widetilde{c}_{3}(t)+\beta e^{-d_{4} \tau_{4}} S\left(t-\tau_{4}\right)\right. \\
& \left.\quad-a_{33} I(t)\right] \mathrm{d} t+\sigma_{3} \mathrm{~d} B_{3}(t) \\
& \quad+\int_{\mathbb{Y}} \ln \left(1+\gamma_{3}(u)\right) \widetilde{N}(\mathrm{~d} t, \mathrm{~d} u) .
\end{aligned}
$$

Then we can obtain

$$
\begin{aligned}
& \frac{\ln X(t)-\ln X(0)}{t}=b_{1}-\delta_{1}\left\langle\widetilde{c}_{1}(t)\right\rangle \\
& -a_{12} e^{-d_{1} \tau_{1}}\langle S(t)\rangle-a_{11}\langle X(t)\rangle-a_{12} e^{-d_{1} \tau_{1}}
\end{aligned}
$$

$$
\begin{aligned}
& \frac{\left(\int_{0}^{\tau_{1}} X\left(s-\tau_{1}\right) \mathrm{d} s-\int_{t}^{t+\tau_{1}} X\left(s-\tau_{1}\right) \mathrm{d} s\right)}{t} \\
& +\frac{\sigma_{1} B_{1}(t)}{t}+\frac{1}{t} \int_{0}^{t} \int_{\mathbb{V}} \ln \left(1+\gamma_{1}(u)\right) \widetilde{N}(\mathrm{~d} s, \mathrm{~d} u),
\end{aligned}
$$

$$
\begin{aligned}
& \frac{\ln S(t)-\ln S(0)}{t}=-b_{2}-\delta_{2}\left\langle\widetilde{c}_{2}(t)\right\rangle \\
& \quad+a_{21} e^{-d_{2} \tau_{2}}\langle X(t)\rangle-\beta e^{-d_{3} \tau_{3}}\langle I(t)\rangle-a_{22}\langle S(t)\rangle \\
& \quad-a_{21} e^{-d_{2} \tau_{2}}
\end{aligned}
$$$$
\frac{\left(\int_{t}^{t+\tau_{2}} X\left(s-\tau_{2}\right) \mathrm{d} s-\int_{0}^{\tau_{2}} X\left(s-\tau_{2}\right) \mathrm{d} s\right)}{t}
$$$$
+\beta e^{-d_{3} \tau_{3}} \frac{\left(\int_{t}^{t+\tau_{2}} I\left(s-\tau_{2}\right) \mathrm{d} s-\int_{0}^{\tau_{2}} I\left(s-\tau_{2}\right) \mathrm{d} s\right)}{t}
$$$$
+\frac{\sigma_{2} B_{2}(t)}{t}+\frac{1}{t} \int_{0}^{t} \int_{\mathbb{Y}} \ln \left(1+\gamma_{2}(u)\right) \widetilde{N}(\mathrm{~d} s, \mathrm{~d} u),
$$$$
\frac{\ln I(t)-\ln I(0)}{t}=-b_{3}-\delta_{3}\left\langle\widetilde{c}_{3}(t)\right\rangle+\beta e^{-d_{4} \tau_{4}}\langle S(t)\rangle
$$$$
-a_{33}\langle I(t)\rangle-\beta e^{-d_{4} \tau_{4}}
$$$$
\frac{\left(\int_{t}^{t+\tau_{4}} S\left(s-\tau_{4}\right) \mathrm{d} s-\int_{0}^{\tau_{4}} S\left(s-\tau_{4}\right) \mathrm{d} s\right)}{t}
$$$$
+\frac{\sigma_{3} B_{3}(t)}{t}+\frac{1}{t} \int_{0}^{t} \int_{\mathbb{Y}} \ln \left(1+\gamma_{3}(u)\right) \widetilde{N}(\mathrm{~d} s, \mathrm{~d} u) .
$$

Computing $(60) \times a_{21} e^{-d_{2} \tau_{2}}+(61) \times a_{11}$ leads to

$$
\begin{aligned}
a_{21} e^{-d_{2} \tau_{2}} \frac{\ln X(t)-\ln X(0)}{t}+a_{11} \frac{\ln S(t)-\ln S(0)}{t} \\
=\left(b_{1}-\delta_{1}\left\langle\widetilde{c}_{1}(t)\right\rangle\right) a_{21} e^{-d_{2} \tau_{2}} \\
\quad-\left(b_{2}+\delta_{2}\left\langle\widetilde{c}_{2}(t)\right\rangle\right) a_{11}-\Delta^{\prime}\langle S(t)\rangle \\
\quad-a_{11} \beta e^{-d_{3} \tau_{3}}\langle I(t)\rangle+\Gamma_{1},
\end{aligned}
$$

where $\Gamma_{1}$ is given in Appendix.

Case (i). By Lemma 5, we know that $\lim _{t \rightarrow \infty} X_{1}(t)=0$, if $b_{1}<$ $\delta_{1} \bar{c}_{1}$. From (53), we have

$$
\lim _{t \rightarrow \infty} X(t)=0 \quad \text { a.s. }
$$

Obviously, we get

$$
\begin{aligned}
& \lim _{t \rightarrow \infty} S(t)=0, \\
& \lim _{t \rightarrow \infty} I(t)=0,
\end{aligned}
$$


Case (ii). By Lemma 5, we know that $\lim _{t \rightarrow \infty} S_{1}(t)=0$, if $b_{1}>$ $\delta_{1} \bar{c}_{1}$ and $\Delta_{1}<0$. From (53), we have

$$
\lim _{t \rightarrow \infty} S(t)=0 \quad \text { a.s. }
$$

Then we obtain

$$
\lim _{t \rightarrow \infty} I(t)=0 \quad \text { a.s. }
$$

By using (60) and Lemma 4, we can prove

$$
\lim _{t \rightarrow \infty}\langle X(t)\rangle=\frac{b_{1}-\delta_{1} \bar{c}_{1}}{a_{11}} \quad \text { a.s. }
$$

Case (iii). Similarly, by Lemma 5, we get that $\lim _{t \rightarrow \infty} I_{1}(t)=0$, if $b_{1}>\delta_{1} \bar{c}_{1}, \Delta_{1}>0$, and $\mathscr{R}<1$. By (53), we obtain

$$
\lim _{t \rightarrow \infty} I(t)=0 \quad \text { a.s. }
$$

Combining Lemma 4 with (56) and (63), we get

$$
\langle S(t)\rangle_{*} \geq \frac{\Delta_{1}}{\Delta^{\prime}} .
$$

From (60), we have

$$
\begin{aligned}
& \frac{\ln X(t)-\ln X(0)}{t} \\
& \leq b_{1}-\delta_{1}\left\langle\widetilde{c}_{1}(t)\right\rangle_{*}-a_{12} e^{-d_{1} \tau_{1}}\langle S(t)\rangle_{*}-a_{11}\langle X(t)\rangle \\
& \quad+\frac{\sigma_{1} B_{1}(t)}{t}+\frac{1}{t} \int_{0}^{t} \int_{\mathbb{Y}} \ln \left(1+\gamma_{1}(u)\right) \widetilde{N}(\mathrm{~d} s, \mathrm{~d} u) .
\end{aligned}
$$

Then we infer from Lemma 4 that

$$
\begin{aligned}
& \langle X(t)\rangle^{*} \\
& \leq \frac{\left(b_{1}-\delta_{1}\left\langle\widetilde{c}_{1}(t)\right\rangle_{*}\right) a_{22}+\left(b_{2}+\delta_{2}\left\langle\widetilde{c}_{2}(t)\right\rangle^{*}\right) a_{12} e^{-d_{1} \tau_{1}}}{\Delta^{\prime}} .
\end{aligned}
$$

By (61), we get

$$
\begin{aligned}
& \frac{\ln S(t)-\ln S(0)}{t} \\
& \leq-b_{2}-\delta_{2}\left\langle\widetilde{\mathcal{c}}_{2}(t)\right\rangle_{*}+a_{21} e^{-d_{2} \tau_{2}}\langle X(t)\rangle^{*} \\
& \quad-a_{22}\langle S(t)\rangle+\frac{\sigma_{2} B_{2}(t)}{t} \\
& \quad+\frac{1}{t} \int_{0}^{t} \int_{\mathbb{Y}} \ln \left(1+\gamma_{2}(u)\right) \widetilde{N}(\mathrm{~d} s, \mathrm{~d} u) .
\end{aligned}
$$

From Lemma 4, we obtain

$$
\langle S(t)\rangle^{*} \leq \frac{\Delta_{1}}{\Delta^{\prime}} .
$$

By (60), we have

$$
\begin{aligned}
& \frac{\ln X(t)-\ln X(0)}{t} \\
& \geq b_{1}-\delta_{1}\left\langle\widetilde{c}_{1}(t)\right\rangle^{*}-a_{12} e^{-d_{1} \tau_{1}}\langle S(t)\rangle^{*} \\
& \quad-a_{11}\langle X(t)\rangle+\frac{\sigma_{1} B_{1}(t)}{t} \\
& \quad+\frac{1}{t} \int_{0}^{t} \int_{\mathbb{Y}} \ln \left(1+\gamma_{1}(u)\right) \widetilde{N}(\mathrm{~d} s, \mathrm{~d} u) .
\end{aligned}
$$

In view of Lemma 4 , we can see that

$$
\begin{aligned}
& \langle X(t)\rangle_{*} \\
& \geq \frac{\left(b_{1}-\delta_{1}\left\langle\widetilde{c}_{1}(t)\right\rangle^{*}\right) a_{22}+\left(b_{2}+\delta_{2}\left\langle\widetilde{c}_{2}(t)\right\rangle_{*}\right) a_{12} e^{-d_{1} \tau_{1}}}{\Delta^{\prime}} .
\end{aligned}
$$

Consequently,

$$
\lim _{t \rightarrow \infty}\langle X(t)\rangle=\frac{\left(b_{1}-\delta_{1} \bar{c}_{1}\right) a_{22}+\left(b_{2}+\delta_{2} \bar{c}_{2}\right) a_{12} e^{-d_{1} \tau_{1}}}{\Delta^{\prime}}
$$

$$
\lim _{t \rightarrow \infty}\langle S(t)\rangle=\frac{\Delta_{1}}{\Delta^{\prime}} \quad \text { a.s. }
$$

This completes the proof of Theorem 6 .

3.2. Permanence in Mean. In this section, we prove the permanence in mean of system (10).

Theorem 7. Let $(X(t), S(t), I(t))$ be the solution of system (10) with initial value $\left(\left(\phi_{1}(t), \phi_{2}(t), \phi_{3}(t)\right):-\tau \leq t \leq\right.$ $0) \in C\left([-\tau, 0] ; R_{+}^{3}\right)$. If $\Delta_{2}>0$ and $\Delta_{3}>0$, when $\widehat{\mathscr{R}}=$ $\left(a_{11} a_{22} / \Delta^{\prime}\right) \mathscr{R}>1$, then

$$
\begin{aligned}
& \lim _{t \rightarrow \infty}\langle X(t)\rangle=\frac{\Delta_{2}}{\Delta}, \\
& \lim _{t \rightarrow \infty}\langle S(t)\rangle=\frac{\Delta_{3}}{\Delta}, \\
& \lim _{t \rightarrow \infty}\langle I(t)\rangle=\frac{\Delta_{4}}{\Delta},
\end{aligned}
$$

a.s.

Proof. Computing $(62) \times \Delta^{\prime}+(63) \times \beta e^{-d_{4} \tau_{4}}$, we obtain

$$
\begin{aligned}
\Delta^{\prime} & \frac{\ln I(t)-\ln I(0)}{t} \\
& +\beta a_{21} e^{-d_{2} \tau_{2}-d_{4} \tau_{4}} \frac{\ln X(t)-\ln X(0)}{t} \\
& +a_{11} \beta e^{-d_{4} \tau_{4}} \frac{\ln S(t)-\ln S(0)}{t} \\
= & -\left(b_{3}+\delta_{3}\left\langle\widetilde{c}_{3}(t)\right\rangle\right) \Delta^{\prime} \\
& +\left(b_{1}-\delta_{1}\left\langle\widetilde{c}_{1}(t)\right\rangle\right) a_{21} \beta e^{-d_{2} \tau_{2}-d_{4} \tau_{4}} \\
& -\left(b_{2}+\delta_{2}\left\langle\widetilde{c}_{2}(t)\right\rangle\right) a_{11} \beta e^{-d_{4} \tau_{4}} \\
& -\left(a_{33} \Delta^{\prime}+a_{11} \beta^{2} e^{-d_{3} \tau_{3}-d_{4} \tau_{4}}\right)\langle I(t)\rangle+\Gamma_{2},
\end{aligned}
$$

where $\Gamma_{2}$ is given in Appendix.

When $\widehat{\mathscr{R}}=\left(a_{11} a_{22} / \Delta^{\prime}\right) \mathscr{R}>1$, by Lemma 4 , we have

$$
\langle I(t)\rangle_{*} \geq \frac{\Delta_{4}}{\Delta} .
$$


So

$$
\lim _{t \rightarrow \infty} \frac{\ln I(t)}{t}=0 \quad \text { a.s. }
$$

Computing $(63) \times a_{33}-(62) \times a_{11} \beta e^{-d_{3} \tau_{3}}$, we have

$$
\begin{aligned}
a_{33} a_{21} e^{-d_{2} \tau_{2}} \frac{\ln X(t)-\ln X(0)}{t} & \\
& +a_{11} a_{33} \frac{\ln S(t)-\ln S(0)}{t} \\
& -a_{11} \beta e^{-d_{3} \tau_{3}} \frac{\ln I(t)-\ln I(0)}{t} \\
= & \left(b_{3}+\delta_{3}\left\langle\widetilde{c}_{3}(t)\right\rangle\right) a_{11} \beta e^{-d_{3} \tau_{3}} \\
& +\left(b_{1}-\delta_{1}\left\langle\widetilde{c}_{1}(t)\right\rangle\right) a_{33} a_{21} e^{-d_{2} \tau_{2}} \\
& -\left(b_{2}+\delta_{2}\left\langle\widetilde{c}_{2}(t)\right\rangle\right) a_{11} a_{33} \\
& -\left(a_{33} \Delta^{\prime}+a_{11} \beta^{2} e^{-d_{3} \tau_{3}-d_{4} \tau_{4}}\right)\langle S(t)\rangle+\Gamma_{3},
\end{aligned}
$$

where $\Gamma_{3}$ is given in Appendix.

When $\Delta_{3}>0$, by Lemma 4 , we have

$$
\langle S(t)\rangle_{*} \geq \frac{\Delta_{3}}{\Delta} .
$$

From (71), when $\Delta_{2}>0$, we obtain

$$
\langle X(t)\rangle^{*} \leq \frac{\Delta_{2}}{\Delta} .
$$

By (61), we get

$$
\begin{aligned}
& \frac{\ln S(t)-\ln S(0)}{t} \\
& \leq-b_{2}-\delta_{2}\left\langle\widetilde{c}_{2}(t)\right\rangle_{*}+a_{21} e^{-d_{2} \tau_{2}}\langle X(t)\rangle^{*} \\
& \quad-\beta e^{-d_{3} \tau_{3}}\langle I(t)\rangle_{*}-a_{22}\langle S(t)\rangle+\frac{\sigma_{2} B_{2}(t)}{t} \\
& \quad+\frac{1}{t} \int_{0}^{t} \int_{\mathbb{Y}} \ln \left(1+\gamma_{2}(u)\right) \widetilde{N}(\mathrm{~d} s, \mathrm{~d} u),
\end{aligned}
$$

then from Lemma 4 we prove that

$$
\langle S(t)\rangle^{*} \leq \frac{\Delta_{3}}{\Delta} .
$$

By (60), we have

$$
\begin{aligned}
& \frac{\ln X(t)-\ln X(0)}{t} \\
& \geq b_{1}-\delta_{1}\left\langle\widetilde{c}_{1}(t)\right\rangle^{*}-a_{12} e^{-d_{1} \tau_{1}}\langle S(t)\rangle^{*} \\
& \quad-a_{11}\langle X(t)\rangle+\frac{\sigma_{1} B_{1}(t)}{t} \\
& \quad+\frac{1}{t} \int_{0}^{t} \int_{\mathbb{Y}} \ln \left(1+\gamma_{1}(u)\right) \widetilde{N}(\mathrm{~d} s, \mathrm{~d} u),
\end{aligned}
$$

and then from Lemma 4 we get

$$
\langle X(t)\rangle_{*} \geq \frac{\Delta_{2}}{\Delta}
$$

It can be inferred from (62) that

$$
\begin{aligned}
& \frac{\ln I(t)-\ln I(0)}{t} \\
& \leq-b_{3}-\delta_{3}\left\langle\widetilde{c}_{3}(t)\right\rangle_{*}+\beta e^{-d_{4} \tau_{4}}\langle S(t)\rangle^{*}-a_{33}\langle I(t)\rangle \\
& \quad-\beta e^{-d_{4} \tau_{4}} \frac{\left(\int_{t}^{t+\tau_{4}} S\left(s-\tau_{4}\right) \mathrm{d} s-\int_{0}^{\tau_{4}} S\left(s-\tau_{4}\right) \mathrm{d} s\right)}{t} \\
& +\frac{\sigma_{3} B_{3}(t)}{t}+\frac{1}{t} \int_{0}^{t} \int_{\mathbb{Y}} \ln \left(1+\gamma_{3}(u)\right) \widetilde{N}(\mathrm{~d} s, \mathrm{~d} u),
\end{aligned}
$$

and then from Lemma 4 we have

$$
\langle I(t)\rangle^{*} \leq \frac{\Delta_{4}}{\Delta} .
$$

Consequently,

$$
\begin{aligned}
& \lim _{t \rightarrow \infty}\langle X(t)\rangle=\frac{\Delta_{2}}{\Delta}, \\
& \lim _{t \rightarrow \infty}\langle S(t)\rangle=\frac{\Delta_{3}}{\Delta}, \\
& \lim _{t \rightarrow \infty}\langle I(t)\rangle=\frac{\Delta_{4}}{\Delta},
\end{aligned}
$$

a.s.

This completes the proof of Theorem 7.

\section{Conclusion and Simulations}

This paper explores the dynamics of a stochastic predatorprey model with time delays in the polluted environment. We show some properties of the subsystem of the predatorprey system. Then by using comparison theorem and limit superior theory, we obtain the sufficient conditions for the extinction of this system. From Theorem 6, we know that if environmental noises are large enough, the species will be extinct (see Figure 1) and if the amount of pulsed input concentration of the toxicant is large enough or the pulse period is small enough, the species will also be extinct (see Figures 2 and 3). In addition, Theorem 7 indicates that the permanence of populations has high correlation with the intensity of environmental noises (see Figure 2(a)). The main results show that time delays can lead to extinction of this system. Therefore, we realize that the environmental noises, the toxicant input, and delays are harmful to the permanence of the populations. Now we show some simulations to verify our main results.

Choose some parameters as follows $r=1.6, \mu_{1}=0.1$, $\mu_{2}=0.1, \delta_{1}=0.3, \delta_{2}=0.1, \delta_{3}=0.1, a_{11}=0.3, a_{12}=0.3$, $a_{21}=0.3, a_{22}=0.3, a_{33}=0.1, d_{1}=0.1, d_{2}=0.1, d_{3}=0.1$, 


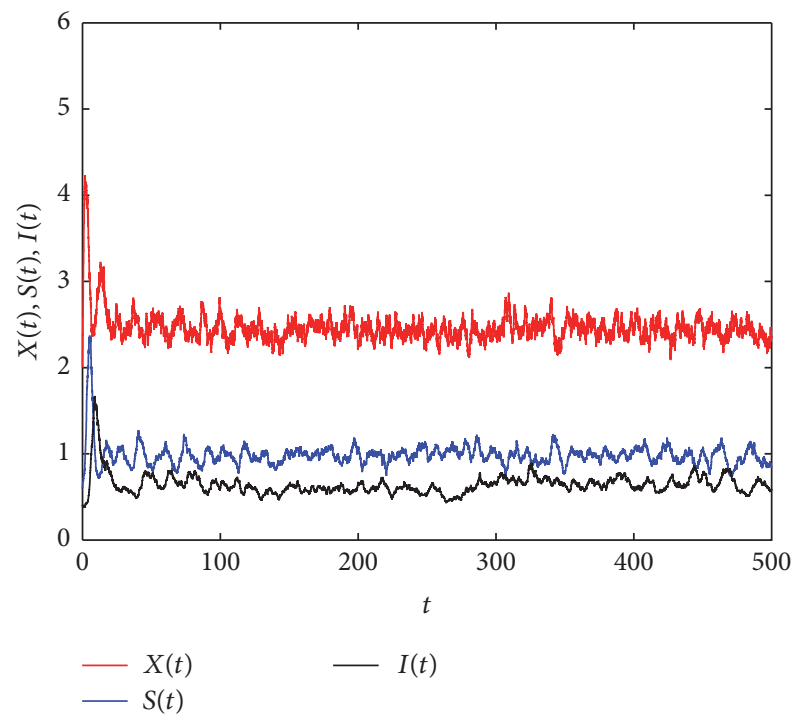

(a)

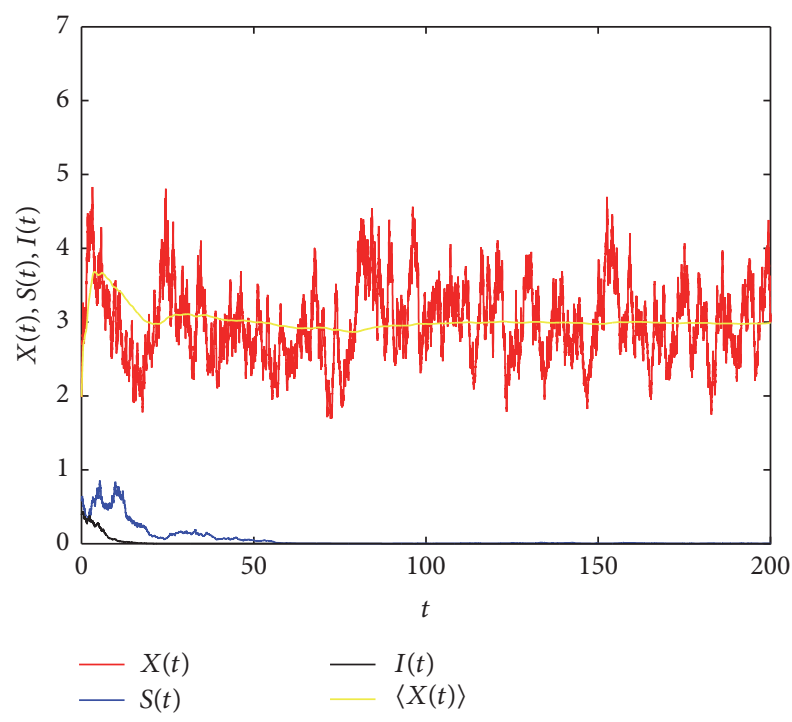

(c)

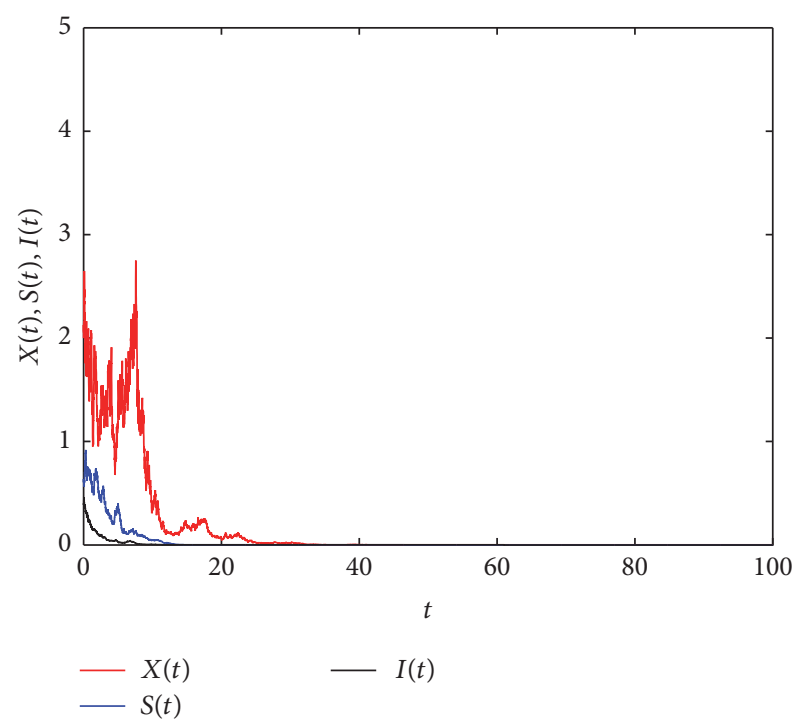

(b)

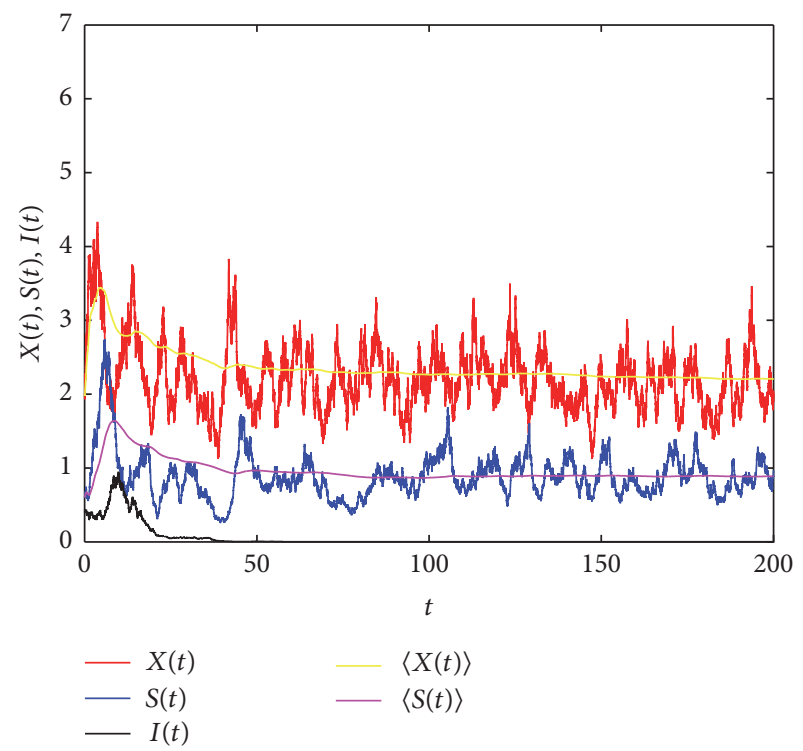

(d)

Figure 1: In (a), $\gamma_{1}=0, \gamma_{2}=0, \gamma_{3}=0$, and the species are permanent; in (b), $\gamma_{1}=2.2, \gamma_{2}=1.5, \gamma_{3}=1.2$, and then the species are extinct; in (c), $\gamma_{1}=0.5, \gamma_{2}=1.5, \gamma_{3}=1.2, X(t)$ is permanent, $S(t)$ and $I(t)$ are extinct, and $\lim _{t \rightarrow \infty}\langle X(t)\rangle=2.953$; in $(\mathrm{d}), \gamma_{1}=0.5, \gamma_{2}=0.5, \gamma_{3}=1.2$, $X(t)$ and $S(t)$ are permanent, $I(t)$ is extinct, $\lim _{t \rightarrow \infty}\langle X(t)\rangle=2.144$, and $\lim _{t \rightarrow \infty}\langle S(t)\rangle=0.892$.

$d_{4}=0.1, \tau_{1}=1, \tau_{2}=1, \tau_{3}=1, \tau_{4}=1, h=0.3, \sigma_{1}=0.2$, $\sigma_{2}=0.2, \sigma_{3}=0.1, k_{1}=0.3, g_{1}=0.1, m_{1}=0.1, k_{2}=0.3$, $g_{2}=0.2, m_{2}=0.2, k_{3}=0.3, g_{3}=0.2$, and $m_{3}=0.2$. Based on the above parameters, we give simulations to explain the biological implications.

In Figure 1, we keep $u=0.4$ and $T=1$. In Figure $1(\mathrm{a})$, choose $\gamma_{1}=0, \gamma_{2}=0$, and $\gamma_{3}=0$, there is no Lévy noises, and we can see that the species are permanent. In Figure 1(b), if $\gamma_{1}=2.2, \gamma_{2}=1.5$, and $\gamma_{3}=1.2$, the intensities of Lévy noises are large and then we get $b_{1}=0.543<\delta_{1} \bar{c}_{1}=0.600$, which means that the productiveness of the prey is less than its death loss rate; from Theorem 6 , the species go extinction. In Figure 1(c), if $\gamma_{1}=0.5, \gamma_{2}=1.5$, and $\gamma_{3}=1.2$, then $b_{1}=$ $0.886>\delta_{1} \bar{c}_{1}=0.600$ and $\Delta_{1}=-0.009<0$, from Theorem 6 we have that $X(t)$ is permanent and $S(t)$ and $I(t)$ are extinct; furthermore, we have $\lim _{t \rightarrow \infty}\langle X(t)\rangle=2.953$. In Figure 1(d), if $\gamma_{1}=0.5, \gamma_{2}=0.5$, and $\gamma_{3}=1.2$, then $b_{1}=0.886>\delta_{1} \bar{c}_{1}=$ $0.600, \Delta_{1}=0.146>0$, and $\mathscr{R}=0.946<1$; from Theorem 6 we obtain that $X(t)$ and $S(t)$ are permanent and $I(t)$ is extinct and we get $\lim _{t \rightarrow \infty}\langle X(t)\rangle=2.144$ and $\lim _{t \rightarrow \infty}\langle S(t)\rangle=0.892$.

In Figure 2, we keep $\gamma_{1}=0.5, \gamma_{2}=0.5, \gamma_{3}=0.1$, and $T=1$. In Figure 2(a), we choose $u=0.4$, and then $\Delta_{2}=$ $0.114>0, \Delta_{3}=0.037>0$, and $\mathscr{R}=2.809>\left(a_{11} a_{22} / \Delta^{\prime}\right) \mathscr{R}=$ $1.559>1$. From Theorem 7, we have that $X(t), S(t)$, and $I(t)$ are permanent and we obtain that $\lim _{t \rightarrow \infty}\langle X(t)\rangle=$ 2.072, $\lim _{t \rightarrow \infty}\langle S(t)\rangle=0.680$ and $\lim _{t \rightarrow \infty}\langle I(t)\rangle=0.345$. In 


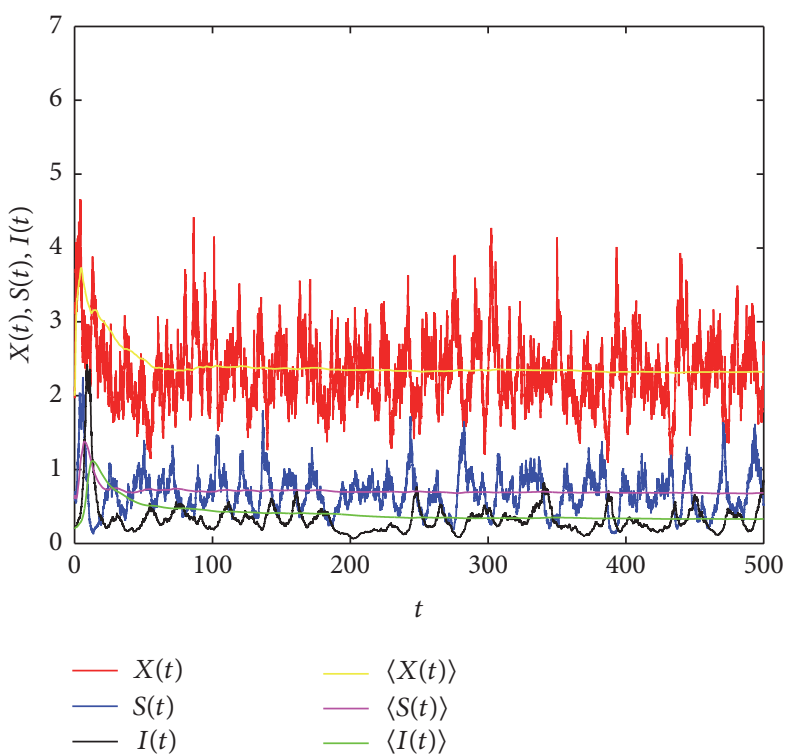

(a)

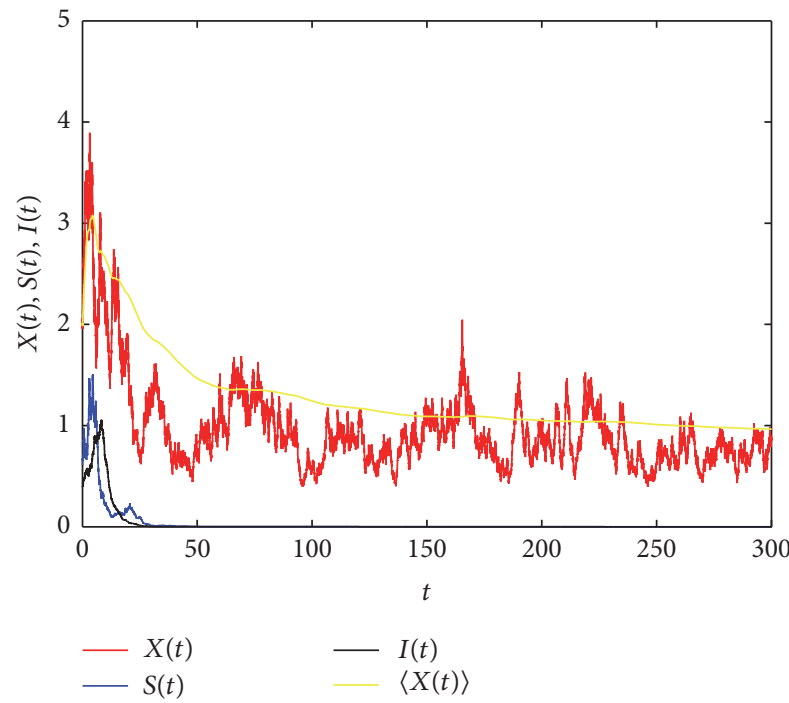

(c)

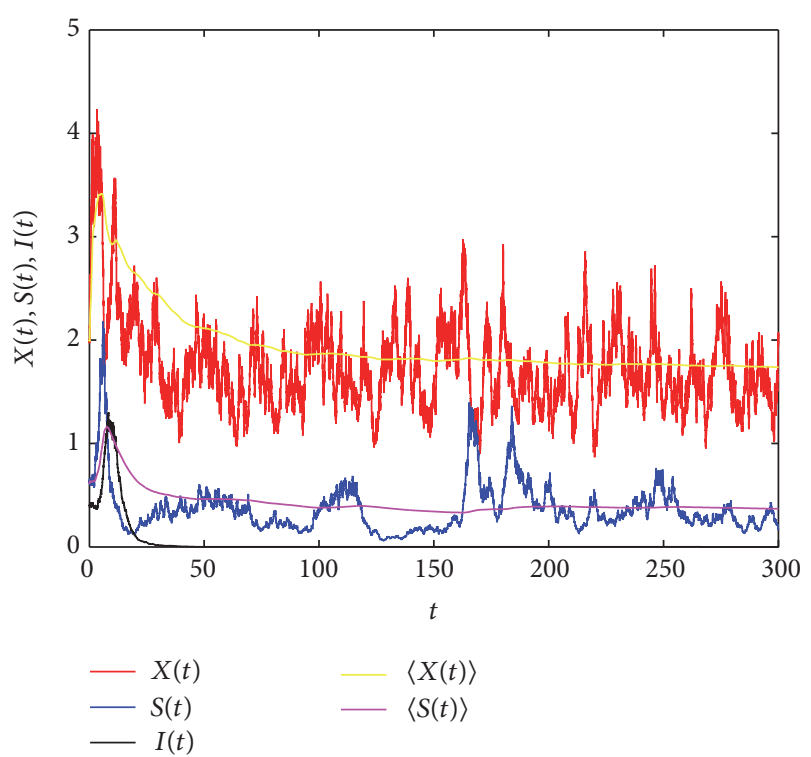

(b)

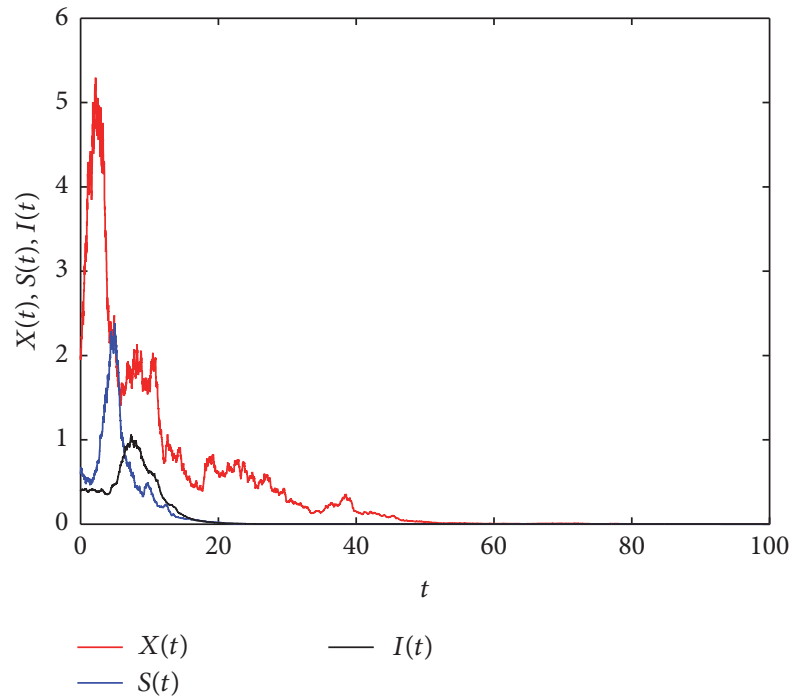

(d)

Figure 2: Keep $\gamma_{1}=0.5, \gamma_{2}=0.5, \gamma_{3}=0.1$, and $T=1$. In (a), $u=0.4, X(t), S(t)$, and $I(t)$ are permanent, $\lim _{t \rightarrow \infty}\langle X(t)\rangle=2.072$, $\lim _{t \rightarrow \infty}\langle S(t)\rangle=0.680$, and $\lim _{t \rightarrow \infty}\langle I(t)\rangle=0.345$; in (b), $u=0.6, X(t)$ and $S(t)$ are permanent, $I(t)$ is extinct, $\lim _{t \rightarrow \infty}\langle X(t)\rangle=1.719$, and $\lim _{t \rightarrow \infty}\langle S(t)\rangle=0.313$; in (c), $u=0.8, X(t)$ is permanent, $S(t)$ and $I(t)$ are extinct, and $\lim _{t \rightarrow \infty}\langle X(t)\rangle=0.952$; in (d), $u=1$ and then the species are extinct.

Figure 2(b), if $u=0.6$, then $\Delta_{1}=0.050>0$ and $\mathscr{R}=$ $0.768<1$, and from Theorem 6 , we get that $X(t)$ and $S(t)$ are permanent and $I(t)$ is extinct and $\lim _{t \rightarrow \infty}\langle X(t)\rangle=1.719$ and $\lim _{t \rightarrow \infty}\langle S(t)\rangle=0.313$. In Figure 2(c), if $u=0.8$, then $\Delta_{1}=-0.047<0, X(t)$ is permanent, $S(t)$ and $I(t)$ are extinct by Theorem 6 , and $\lim _{t \rightarrow \infty}\langle X(t)\rangle=0.952$. In Figure $2(\mathrm{~d})$, we choose $u=1$, and then $b_{1}=1.4855<\delta_{1} \bar{c}_{1}=1.5$; from Theorem 6 the species are extinct.

In Figure 3, we keep $\gamma_{1}=0.5, \gamma_{2}=0.5, \gamma_{3}=0.1$, and $u=0.4$. In Figure 3(a), we choose $T=1$ and then $\Delta_{2}=$ $0.114>0, \Delta_{3}=0.037>0$, and $\mathscr{R}=2.809>\left(a_{11} a_{22} / \Delta^{\prime}\right) \mathscr{R}=$ $1.559>1$. From Theorem 7, we have that $X(t), S(t)$, and
$I(t)$ are permanent and we obtain that $\lim _{t \rightarrow \infty}\langle X(t)\rangle=$ 2.072, $\lim _{t \rightarrow \infty}\langle S(t)\rangle=0.680$, and $\lim _{t \rightarrow \infty}\langle I(t)\rangle=0.345$. In Figure 3(b), if $T=0.7$, then $\Delta_{1}=0.063>0$ and $\mathscr{R}=0.710<1$, and from Theorem 6 , we get that $X(t)$ and $S(t)$ are permanent and $I(t)$ is extinct and $\lim _{t \rightarrow \infty}\langle X(t)\rangle=1.743$ and $\lim _{t \rightarrow \infty}\langle S(t)\rangle=0.386$. In Figure 3(c), if $T=0.5$, then $\Delta_{1}=-0.047<0, X(t)$ is permanent, $S(t)$ and $I(t)$ are extinct by Theorem 6, and $\lim _{t \rightarrow \infty}\langle X(t)\rangle=0.952$. In Figure 3(d), we choose $T=0.4$ and then $b_{1}=1.4855<\delta_{1} \bar{c}_{1}=1.5$; from Theorem 6 the species are extinct.

From Figures 1-3, we can obtain the following conclusions: 


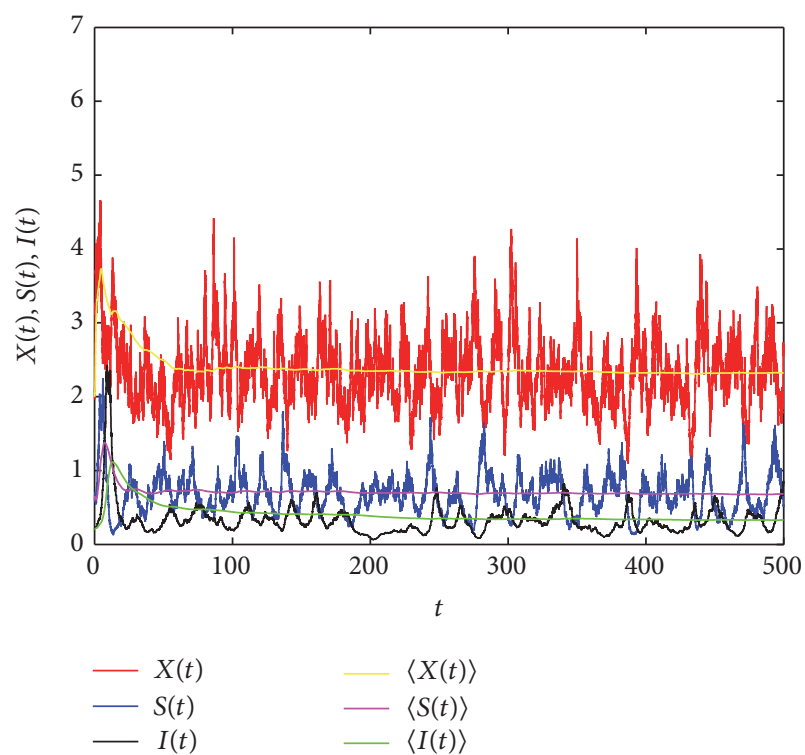

(a)

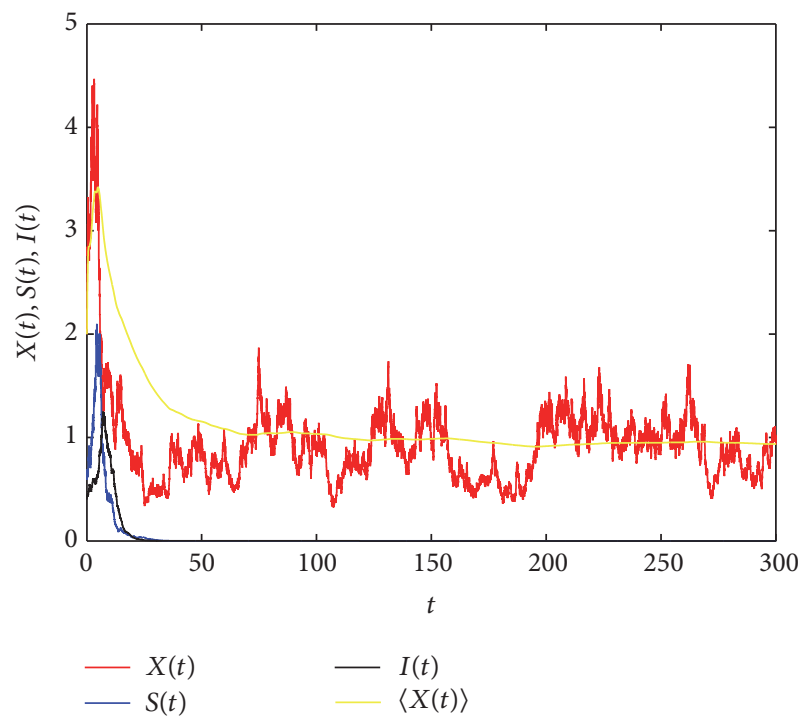

(c)

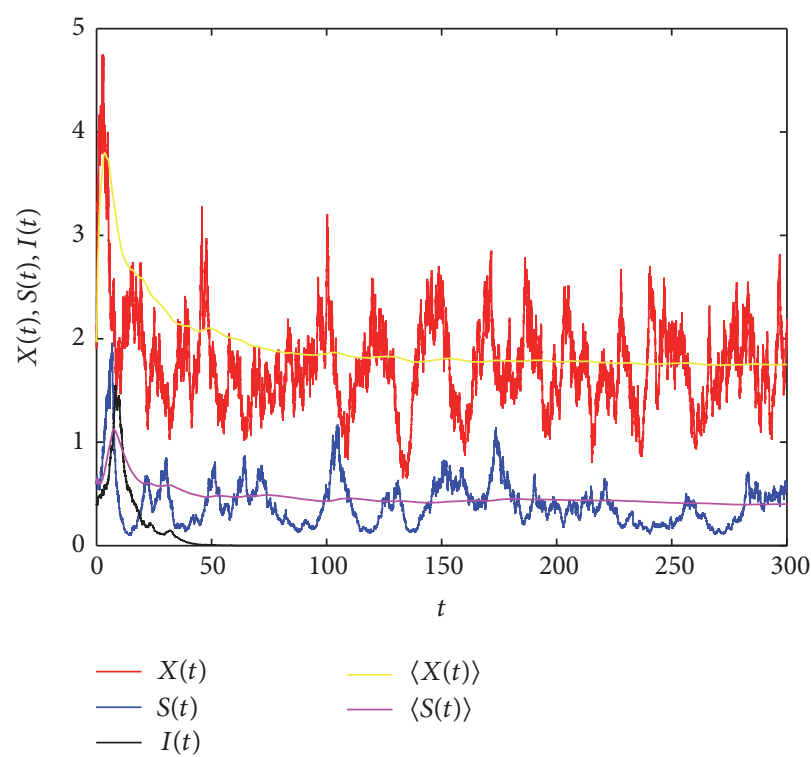

(b)

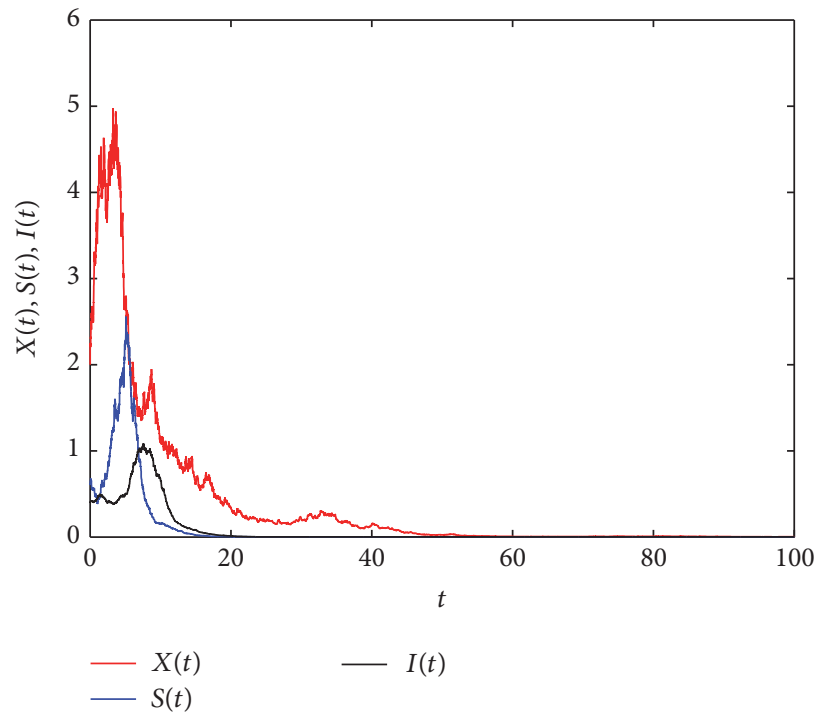

(d)

FIGURE 3: Keep $\gamma_{1}=0.5, \gamma_{2}=0.5, \gamma_{3}=0.1$, and $u=0.4$. In (a), $T=1, X(t), S(t)$ and $I(t)$ are permanent, $\lim _{t \rightarrow \infty}\langle X(t)\rangle=2.072$, $\lim _{t \rightarrow \infty}\langle S(t)\rangle=0.680$, and $\lim _{t \rightarrow \infty}\langle I(t)\rangle=0.345$; in (b), $T=0.7, X(t)$ and $S(t)$ are permanent, $I(t)$ is extinct, $\lim _{t \rightarrow \infty}\langle X(t)\rangle=1.743$, and $\lim _{t \rightarrow \infty}\langle S(t)\rangle=0.386$; in (c), $T=0.5, X(t)$ is permanent, $S(t)$ and $I(t)$ are extinct, and $\lim _{t \rightarrow \infty}\langle X(t)\rangle=0.952$; in (d), $u=0.4$ and then the species are extinct.

(1) Large stochastic disturbance can cause the populations to go to extinction; that is, the persistent population of a deterministic system can become extinct due to the white noise stochastic disturbance.

(2) Large impulsive input concentration of the toxicant or small impulsive period of the exogenous input of toxicant can cause the populations to go to extinction.

Therefore, the above numerical simulations illustrate the performance of the theoretical results, and the biological results show that the white noise stochastic disturbance and impulsive toxicant input are disadvantage for the permanence of system.

\section{Appendix}

For the sake of convenience, we define

$$
\begin{aligned}
\Gamma_{1}= & -a_{12} a_{21} e^{-d_{1} \tau_{1}-d_{2} \tau_{2}} \\
& \cdot \frac{\left(\int_{0}^{\tau_{1}} X\left(s-\tau_{1}\right) \mathrm{d} s-\int_{t}^{t+\tau_{1}} X\left(s-\tau_{1}\right) \mathrm{d} s\right)}{t}
\end{aligned}
$$




$$
\begin{aligned}
& -a_{11} a_{21} e^{-d_{2} \tau_{2}} \\
& \cdot \frac{\left(\int_{t}^{t+\tau_{2}} X\left(s-\tau_{2}\right) \mathrm{d} s-\int_{0}^{\tau_{2}} X\left(s-\tau_{2}\right) \mathrm{d} s\right)}{t} \\
& +a_{11} \beta e^{-d_{3} \tau_{3}} \\
& \cdot \frac{\left(\int_{t}^{t+\tau_{3}} I\left(s-\tau_{3}\right) \mathrm{d} s-\int_{0}^{\tau_{3}} I\left(s-\tau_{3}\right) \mathrm{d} s\right)}{t} \\
& +a_{21} e^{-d_{2} \tau_{2}}\left(\frac{\sigma_{1} B_{1}(t)}{t}\right. \\
& \left.+\frac{1}{t} \int_{0}^{t} \int_{\mathbb{Y}} \ln \left(1+\gamma_{1}(u)\right) \widetilde{N}(\mathrm{~d} s, \mathrm{~d} u)\right) \\
& +a_{11}\left(\frac{\sigma_{2} B_{2}(t)}{t}\right. \\
& \left.+\frac{1}{t} \int_{0}^{t} \int_{\mho} \ln \left(1+\gamma_{2}(u)\right) \widetilde{N}(\mathrm{~d} s, \mathrm{~d} u)\right), \\
& \Gamma_{2}=-\beta a_{12} a_{21} e^{-d_{1} \tau_{1}-d_{2} \tau_{2}-d_{4} \tau_{4}} \\
& \frac{\left(\int_{0}^{\tau_{1}} X\left(s-\tau_{1}\right) \mathrm{d} s-\int_{t}^{t+\tau_{1}} X\left(s-\tau_{1}\right) \mathrm{d} s\right)}{t} \\
& -a_{11} a_{21} \beta e^{-d_{2} \tau_{2}-d_{4} \tau_{4}} \\
& \frac{\left(\int_{t}^{t+\tau_{2}} X\left(s-\tau_{2}\right) \mathrm{d} s-\int_{0}^{\tau_{2}} X\left(s-\tau_{2}\right) \mathrm{d} s\right)}{t} \\
& +a_{11} \beta^{2} e^{-d_{3} \tau_{3}-d_{4} \tau_{4}} \\
& \cdot \frac{\left(\int_{t}^{t+\tau_{3}} I\left(s-\tau_{3}\right) \mathrm{d} s-\int_{0}^{\tau_{3}} I\left(s-\tau_{3}\right) \mathrm{d} s\right)}{t} \\
& -\Delta^{\prime} \beta e^{-d_{4} \tau_{4}} \\
& \cdot \frac{\left(\int_{t}^{t+\tau_{4}} S\left(s-\tau_{4}\right) \mathrm{d} s-\int_{0}^{\tau_{4}} S\left(s-\tau_{4}\right) \mathrm{d} s\right)}{t} \\
& +a_{21} \beta e^{-d_{2} \tau_{2}-d_{4} \tau_{4}}\left(\frac{\sigma_{1} B_{1}(t)}{t}\right. \\
& \left.+\frac{1}{t} \int_{0}^{t} \int_{\mathbb{Y}} \ln \left(1+\gamma_{1}(u)\right) \widetilde{N}(\mathrm{~d} s, \mathrm{~d} u)\right) \\
& +a_{11} \beta e^{-d_{4} \tau_{4}}\left(\frac{\sigma_{2} B_{2}(t)}{t}\right. \\
& \left.+\frac{1}{t} \int_{0}^{t} \int_{\mathbb{V}} \ln \left(1+\gamma_{2}(u)\right) \widetilde{N}(\mathrm{~d} s, \mathrm{~d} u)\right) \\
& +\Delta^{\prime}\left(\frac{\sigma_{3} B_{3}(t)}{t}\right. \\
& \left.+\frac{1}{t} \int_{0}^{t} \int_{\mho} \ln \left(1+\gamma_{3}(u)\right) \widetilde{N}(\mathrm{~d} s, \mathrm{~d} u)\right),
\end{aligned}
$$

$$
\begin{aligned}
\Gamma_{3}= & -a_{33} a_{12} a_{21} e^{-d_{1} \tau_{1}-d_{2} \tau_{2}-d_{4} \tau_{4}} \\
& \cdot \frac{\left(\int_{0}^{\tau_{1}} X\left(s-\tau_{1}\right) \mathrm{d} s-\int_{t}^{t+\tau_{1}} X\left(s-\tau_{1}\right) \mathrm{d} s\right)}{t} \\
& -a_{11} a_{33} a_{21} e^{-d_{2} \tau_{2}} \\
& . \frac{\left(\int_{t}^{t+\tau_{2}} X\left(s-\tau_{2}\right) \mathrm{d} s-\int_{0}^{\tau_{2}} X\left(s-\tau_{2}\right) \mathrm{d} s\right)}{t} \\
& +a_{11} a_{33} \beta e^{-d_{3} \tau_{3}} \\
& . \frac{\left(\int_{t}^{t+\tau_{3}} I\left(s-\tau_{3}\right) \mathrm{d} s-\int_{0}^{\tau_{3}} I\left(s-\tau_{3}\right) \mathrm{d} s\right)}{t} \\
& +a_{11} \beta^{2} e^{-d_{3} \tau_{3}-d_{4} \tau_{4}} \\
& . \frac{\left(\int_{t}^{t+\tau_{4}} S\left(s-\tau_{4}\right) \mathrm{d} s-\int_{0}^{\tau_{4}} S\left(s-\tau_{4}\right) \mathrm{d} s\right)}{t} \\
& +a_{33} a_{21} \beta e^{-d_{2} \tau_{2}}\left(\frac{\sigma_{1} B_{1}(t)}{t}\right. \\
& \left.+\frac{1}{t} \int_{0}^{t} \int_{\mathbb{Y}}^{t} \ln \left(1+\gamma_{3}(u)\right) \widetilde{N}(\mathrm{~d} s, \mathrm{~d} u)\right) . \\
& \left.+\frac{1}{t} \int_{0}^{t} \int_{0}^{t} \int_{\mathbb{Y}} \ln \left(1+\gamma_{1}(u)\right) \widetilde{N}(\mathrm{~d} s, \mathrm{~d} u)\right) \\
& +a_{11} a_{33}\left(\frac{\sigma_{2} B_{2}(t)}{t}\right. \\
&
\end{aligned}
$$

\section{Conflicts of Interest}

The authors declare that there are no conflicts of interest regarding the publication of this paper.

\section{Acknowledgments}

This work was supported by the National Natural Science Foundation of China (11371230 and 11561004), the SDUST Research Fund (2014TDJH102), Joint Innovative Center for Safe and Effective Mining Technology and Equipment of Coal Resources, Shandong Province, the Open Foundation of the Key Laboratory of Jiangxi Province for Numerical Simulation and Emulation Techniques, Gannan Normal University, China, and Shandong Provincial Natural Science Foundation, China (ZR2015AQ001). 


\section{References}

[1] M. Zhien and C. Guirong, "Persistence and extinction of a population in a polluted environment," Mathematical Biosciences, vol. 101, no. 1, pp. 75-97, 1990.

[2] B. Dubey and J. Hussain, "Modelling the interaction of two biological species in a polluted environment," Journal of Mathematical Analysis and Applications, vol. 246, no. 1, pp. 58-79, 2000.

[3] X. Meng, L. Wang, and T. Zhang, "Global dynamics analysis of a nonlinear impulsive stochastic chemostat system in a polluted environment," Journal of Applied Analysis and Computation, vol. 6, no. 3, pp. 865-875, 2016.

[4] S. Sinha, O. P. Misra, and J. Dhar, "Modelling a predator-prey system with infected prey in polluted environment," Applied Mathematical Modelling, vol. 34, no. 7, pp. 1861-1872, 2010.

[5] M. Haque, "A predator-prey model with disease in the predator species only," Nonlinear Analysis. Real World Applications, vol. 11, no. 4, pp. 2224-2236, 2010.

[6] Y. Xiao and L. Chen, "Modeling and analysis of a predator-prey model with disease in the prey," Mathematical Biosciences, vol. 171, no. 1, pp. 59-82, 2001.

[7] T. Feng, X. Meng, L. Liu, and S. Gao, "Application of inequalities technique to dynamics analysis of a stochastic ecoepidemiology model," Journal of Inequalities and Applications, 2016:327, 29 pages, 2016.

[8] T. Zhang, X. Meng, Y. Song, and T. Zhang, "A stage-structured predator-prey SI model with disease in the prey and impulsive effects," Mathematical Modelling and Analysis, vol. 18, no. 4, pp. 505-528, 2013.

[9] D. Mukherjee, "Persistence and global stability of a population in a polluted environment with delay," Journal of Biological Systems, vol. 10, no. 3, pp. 225-232, 2002.

[10] T. Zhang, X. Meng, and T. Zhang, "Global analysis for a delayed SIV model with direct and environmental transmissions," Journal of Applied Analysis and Computation, vol. 6, no. 2, pp. 479491, 2016.

[11] Q. Han, D. Jiang, and C. Ji, "Analysis of a delayed stochastic predator-prey model in a polluted environment," Applied Mathematical Modelling, vol. 38, no. 13, pp. 3067-3080, 2014.

[12] L. Liu and X. Meng, "Optimal harvesting control and dynamics of two-species stochastic model with delays," Advances in Difference Equations, 2017:18, 17 pages, 2017.

[13] W. Zhao, T. Zhang, Z. Chang, X. Meng, and Y. Liu, "Dynamical analysis of SIR epidemic models with distributed delay," Journal of Applied Mathematics, vol. 2013, Article ID 154387, 15 pages, 2013.

[14] H. Cheng and T. Zhang, "A new predator-prey model with a profitless delay of digestion and impulsive perturbation on the prey," Applied Mathematics and Computation, vol. 217, no. 22, pp. 9198-9208, 2011.

[15] R. May, Stability and Complexity in Model Ecosystems, Princeton University Press, NJ, USA, 2001.

[16] D. Zhao and S. Yuan, "Dynamics of the stochastic Leslie-Gower predator-prey system with randomized intrinsic growth rate," Physica A. Statistical Mechanics and its Applications, vol. 461, pp. 419-428, 2016.

[17] S. Li and X. Wang, "Analysis of a stochastic predator-prey model with disease in the predator and Beddington-DeAngelis functional response," Advances in Difference Equations, 2015:224, 21 pages, 2015.
[18] X.-z. Meng, "Stability of a novel stochastic epidemic model with double epidemic hypothesis," Applied Mathematics and Computation, vol. 217, no. 2, pp. 506-515, 2010.

[19] H.-j. Ma, "A separation theorem for stochastic singular linear quadratic control problem with partial information," Acta Mathematicae Applicatae Sinica. English Series, vol. 29, no. 2, pp. 303-314, 2013.

[20] M. Liu, H. Qiu, and K. Wang, "A remark on a stochastic predator-prey system with time delays," Applied Mathematics Letters, vol. 26, no. 3, pp. 318-323, 2013.

[21] R. Rudnicki and K. Pichór, "Influence of stochastic perturbation on prey-predator systems," Mathematical Biosciences, vol. 206, no. 1, pp. 108-119, 2007.

[22] X. Meng, S. Zhao, T. Feng, and T. Zhang, "Dynamics of a novel nonlinear stochastic SIS epidemic model with double epidemic hypothesis," Journal of Mathematical Analysis and Applications, vol. 433, no. 1, pp. 227-242, 2016.

[23] X. Liu, Y. Li, and W. Zhang, "Stochastic linear quadratic optimal control with constraint for discrete-time systems," Applied Mathematics and Computation, vol. 228, pp. 264-270, 2014.

[24] X. Li, X. Lin, and Y. Lin, "Lyapunov-type conditions and stochastic differential equations driven by G-Brownian motion," Journal of Mathematical Analysis and Applications, vol. 439, no. 1, pp. 235-255, 2016.

[25] H. Ma and Y. Jia, "Stability analysis for stochastic differential equations with infinite Markovian switchings," Journal of Mathematical Analysis and Applications, vol. 435, no. 1, pp. 593-605, 2016.

[26] Q. Liu, L. Zu, and D. Jiang, "Dynamics of stochastic predatorprey models with Holling II functional response," Communications in Nonlinear Science and Numerical Simulation, vol. 37, pp. 62-76, 2016.

[27] Q. Liu, D. Jiang, N. Shi, T. Hayat, and A. Alsaedi, "Stochastic mutualism model with Levy jumps," Communications in Nonlinear Science and Numerical Simulation, vol. 43, pp. 78-90, 2017.

[28] J. Bao and C. Yuan, "Stochastic population dynamics driven by Lévy noise," Journal of Mathematical Analysis and Applications, vol. 391, no. 2, pp. 363-375, 2012.

[29] M. Liu and K. Wang, "Stochastic Lotka-Volterra systems with Lévy noise," Journal of Mathematical Analysis and Applications, vol. 410, no. 2, pp. 750-763, 2014.

[30] L. Bai, J. Li, K. Zhang, and W. Zhao, "Analysis of a stochastic ratio-dependent predator-prey model driven by Lévy noise," Applied Mathematics and Computation, vol. 233, pp. 480-493, 2014.

[31] X. Zhang, W. Li, M. Liu, and K. Wang, "Dynamics of a stochastic Holling II one-predator two-prey system with jumps," Physica A. Statistical Mechanics and its Applications, vol. 421, pp. 571582, 2015. 


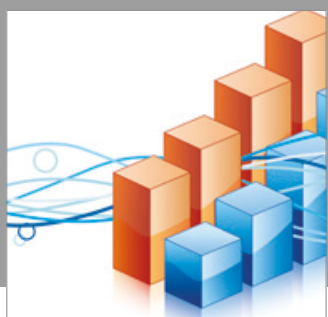

Advances in

Operations Research

vatersals

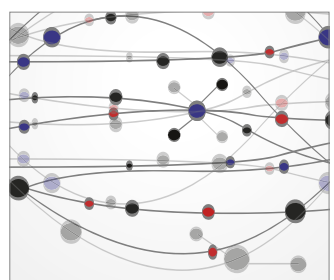

\section{The Scientific} World Journal
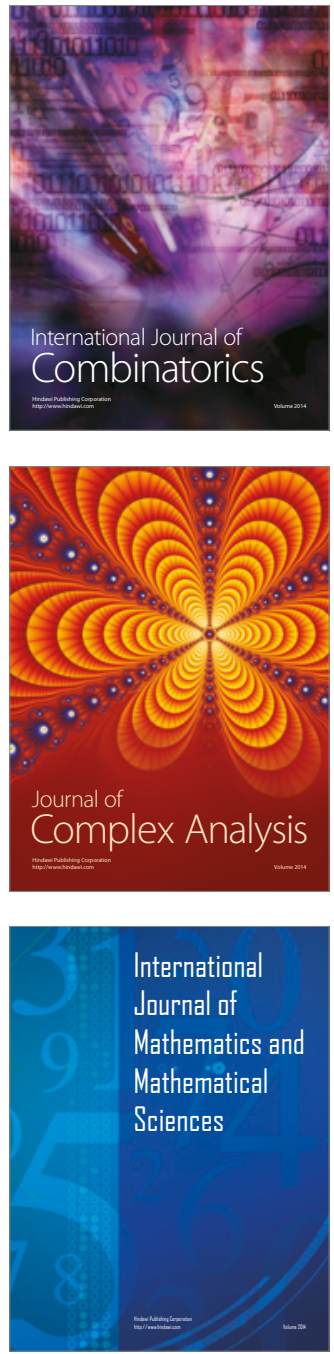
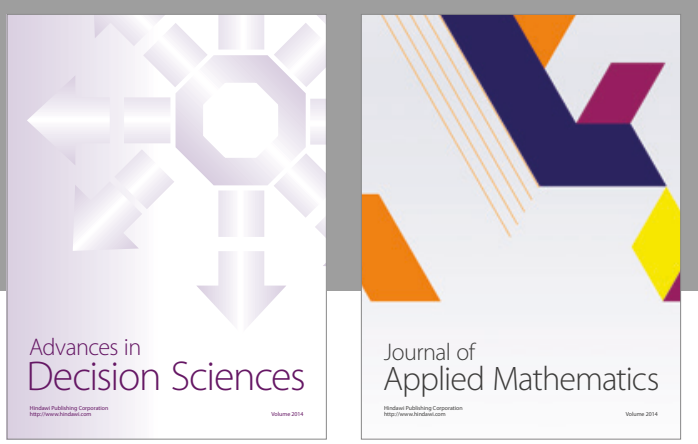

Algebra

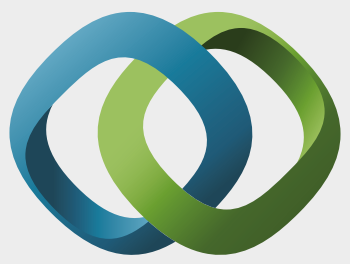

\section{Hindawi}

Submit your manuscripts at

https://www.hindawi.com
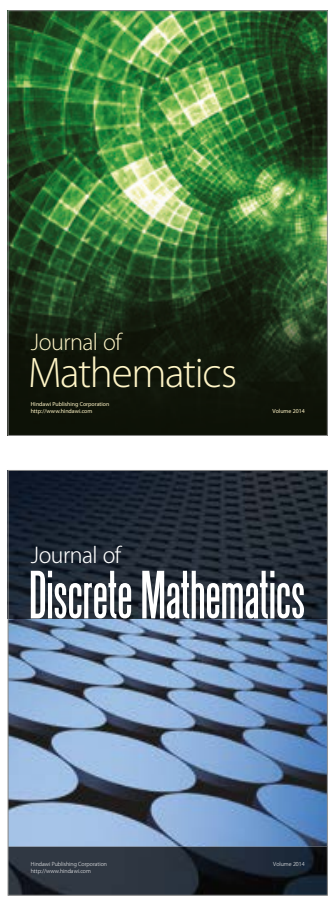

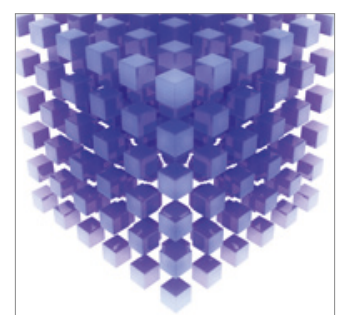

Mathematical Problems in Engineering
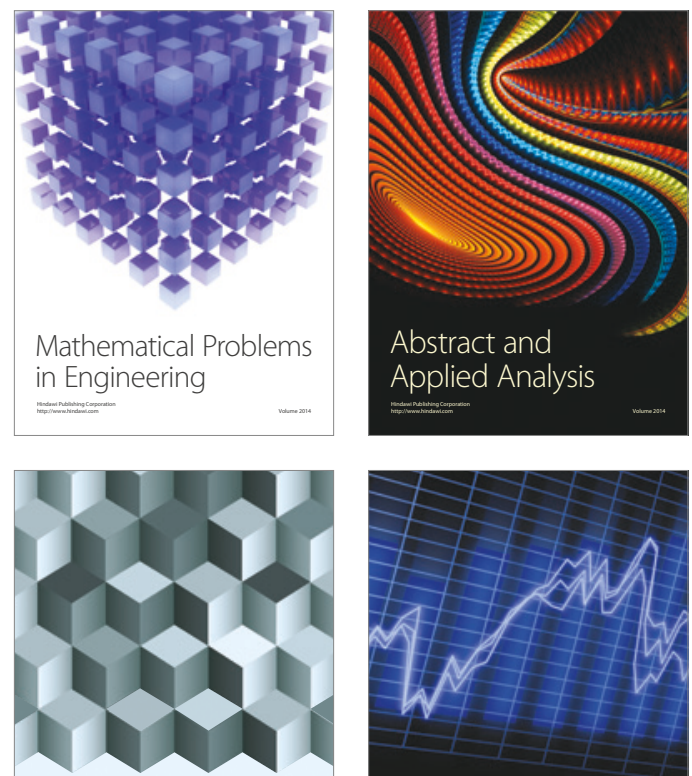

Journal of

Function Spaces

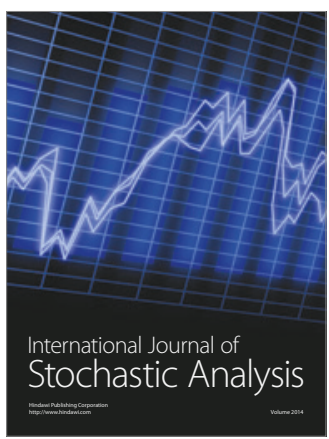

Probability and Statistics
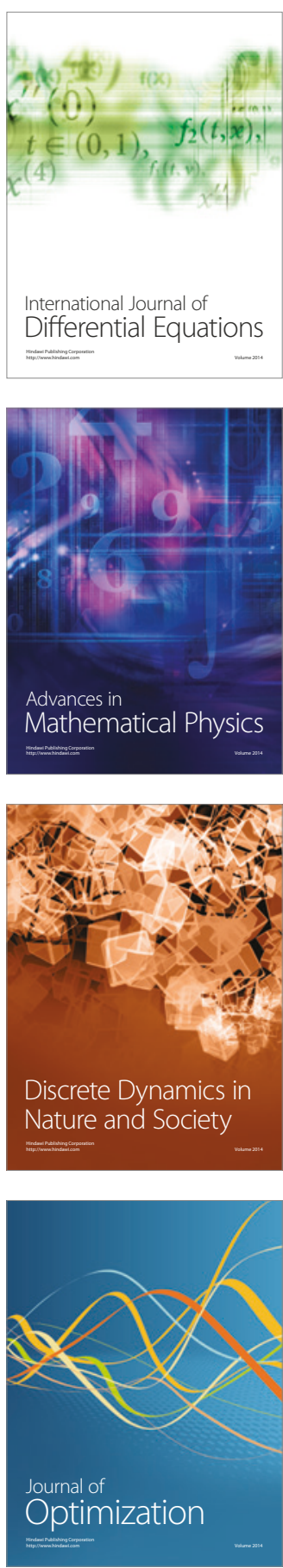